\title{
DES ÉPÉES ROMAINES DANS LA COLLECTION D’ ALISE-SAINTE-REINE
}

\author{
POR
}

ANDRÉ RAPIN

\section{RÉSUMÉ - RESUMEN}

La collection des armes recueillies lors des fouilles d'Alise-Sainte-Reine, commanditées par Napoleon III représente un corpus héterogène. Certaines épées celtiques sont en effet antérieures de plusierus siècles à l'évènement militaire de -52 et d'autres sont en revanche non celtiques.

Deux d'entre elles, soudées par la corrosion et ligaturées par la tôle de bronze d'un fourreau laténien présentent des caractéristiques morphologiques singulieres. Leur analyse technologique permet de les situer dans l'ascendance de la famille des gladii romain. Une première approche comparative en ferait des candidats vraisemblables pour illustrer le texte de Polybe sur le gladius hispaniensis soit, des armes républicaines bien antérieures au siège d'Alésia.

La colección de armas recuperadas en las excavaciones de Alise-Sainte-Reine, impulsadas por Napoleón III, constituye un corpus heterogéneo. Algunas espadas célticas, en efecto, son varios siglos anteriores a los sucesos militares del 52 a.C. e incluso otras no son ni siquiera célticas. En concreto dos de ellas, soldadas por la corrosión y unidas por la lámina broncínea de una vaina de La Tène presentan características morfológicas peculiares. Su análisis tecnológico permite situarlas entre los antecesores de la familia de las espadas romanas. Una primera aproximación comparativa permite considerarlas como candidatos verosímiles para ilustrar el texto de Polibio sobre el gladius hispaniensis, esto es, armas republicanas muy anteriores al asedio de Alesia.

\section{MOTS CLÉS - PALABRAS CLAVE}

Armement romain. Armement celtique. Epées. Gladius hispaniensis.

Armamento romano. Armamento celtico. Espadas. Gladius hispaniensis.

Dans sa récente synthèse sur «Les armes des Romains», Michel Feugère évoque le «problème irritant» posé par l'identification de l'épée romaine d'époque républicaine (Feugère 1993). Le gladius qualifié d'hispaniensis par Polybe n'est, en effet, toujours pas archéologiquement défini. Les descriptions précises qui nous font défaut aujourd'hui n'ont guère préoccupé les historiens antiques et César lui-même ne s'est pas plus attardé à décrire l'épée gauloise que celle de ses légionnaires. A cette indigence documentaire des textes s'ajoute celle d'un corpus d'épées romaines si réduit qu'il nous prive de repères morphologiques. Ainsi, «...l'impossibilité d'identifier la moindre épée romaine dans les fouilles d'Alésia...» est déplorée par M. Feugère dans le même ouvrage (Feugère 1993, p. 97).

La recherche d'éventuels glaives perdus par les vainqueurs d'Alésia serait-elle condamnée à l'échec?

Le fait est que l'identité globale des épées d'Alésia a longtemps été très fluctuante. Romaines tout d'abord, elles deviennent gauloises sous l'impulsion de Verchère de Reffye 
(R.A. 1864, p. 349) (fig. 1). Confirmé un demi-siècle plus tard par J. Déchelette leur statut est à nouveau remis en cause lors des analyses effectuées par P. Couissin qui les identifie à nouveau comme des gladii fabriqués en Gaule par des artisans gaulois (Couissin 1926) (Déchelette 1927). Or, même à l'heure actuelle, nombre d'identifications hâtives de gladii relèvent encore de ce type d'actes de foi idéologiques plus que d'une analyse morphologique précise des documents.

En dehors de quelques bricolages, démontages et remontages, parfois abusifs, contemporains de son dépôt au Musée des Antiquités Nationales, la collection d'Alise s'est peu à peu figée dans les vitrines et dans les réserves. Pendant plus d'un siècle aucune restauration ni analyse radiographique n'est venue renouveler les observations et les premiers relevés du $\mathrm{XIX}^{\mathrm{e}}$ s. L'opportunité d'interrompre cette longue léthargie fût offerte par l'exposition de 1994, Vercingétorix et Alésia ${ }^{1}$. La campagne de restauration programmée pour répondre aux besoins muséographiques était l'occasion d'un examen exhaustif de ce qui avait survécu de l'ensemble quelque peu hétérogène provenant d'Alise-Sainte-Reine.

En effet, parmi la vingtaine d'épées exhumées lors des sondages figurent des armes très anachroniques en regard de l'armement des gaulois de La Tène finale (Pl. II). De plus dans la «récolte» issue des fouilles du XIX ${ }^{\mathrm{e}}$ s. figurent aussi bien des armes des phases anciennes et moyennes de La Tène que des épées non celtiques (Pl. I). Les deux exemples les plus évidents sont publiés pour la première fois avec les quatre planches photographiques qui illustrent la présentation de la collection dans son état initial (Pl. II) (A. Duval 1994). La singularité de ces deux épées est manifeste ne serait-ce que par l'absence originelle du fourreau métallique attestée par la «soudure» des lames provoquée par la corrosion. La ligature de leur extrémité effilée à l'aide d'une tôle de bronze ajoute au caractère insolite de l'ensemble. C'est peut-être ce qui leur a valu d'être tenues à l'écart de toute analyse depuis leur découverte. Furtivement signalées dans le catalogue de l'exposition (Sievers 1994) ainsi que dans un ouvrage de synthèse sur l'armée romaine, elles ne sont toujours pas identifiées (Sievers 1996).

Malgré les efforts déployés par les spécialistes de l'armement romain, la collection d'Alise-Sainte-Reine conserve ainsi sa zone d'ombre injustifiée. La dixième table ronde de la ROMEC réunie en 1996 à Montpellier en témoigne dans ses actes, édités en 1997 par Michel Feugère: les épées d'Alésia sont une fois de plus absentes du débat (Sievers 1997).

La publication des observations et des analyses effectuées lors de leur restauration en 1994, m'a paru d'autant plus indispensable qu'à l'inverse du corpus des épées romaines, celui des épées gauloises comprend plusieurs milliers d'individus. Les grilles d'identifications élaborées depuis deux décennies lors de leurs analyses constituent un bon outil de sélection pour la mise en évidence des armes allogènes. Ce premier tri peut compenser partiellement les carences de la documentation romaine. La recherche du gladius républicain devrait y gagner en efficacité.

\section{SOURCES HISTORIQUES ET CRITÈRES TRADITIONNELS DE DÉTERMINATION}

Selon Polybe, l'arme de poing des fantassins romains du milieu du $\mathrm{II}^{\mathrm{e}}$ s. av. J.-C. est «...un glaive qui se porte sur la cuisse droite et que l'on appelle espagnol. Il a une pointe remarquable et il peut frapper de taille des deux côtés parce que sa lame est forte et solide» (traduction P. Villard. Feugère 1993, p. 80).

1 Vercingétorix et Alésia, exposition organisée par la Réunion des Musées Nationaux et le Musée des Antiquités Nationales du 29 Mars au 18 Juillet 1994. Paris 1994.

Les deux armes analysées dans le présent article ne figuraient pas dans la sélection initiale des objets à restaurer. Elles ont été intégrées à la demande de l'auteur dans le lot des objets prioritaires du fait de leur intérêt archéologique et de leur délabrement (restauration IRRAP, $\mathrm{n}^{\circ}$ 90.42.57). 


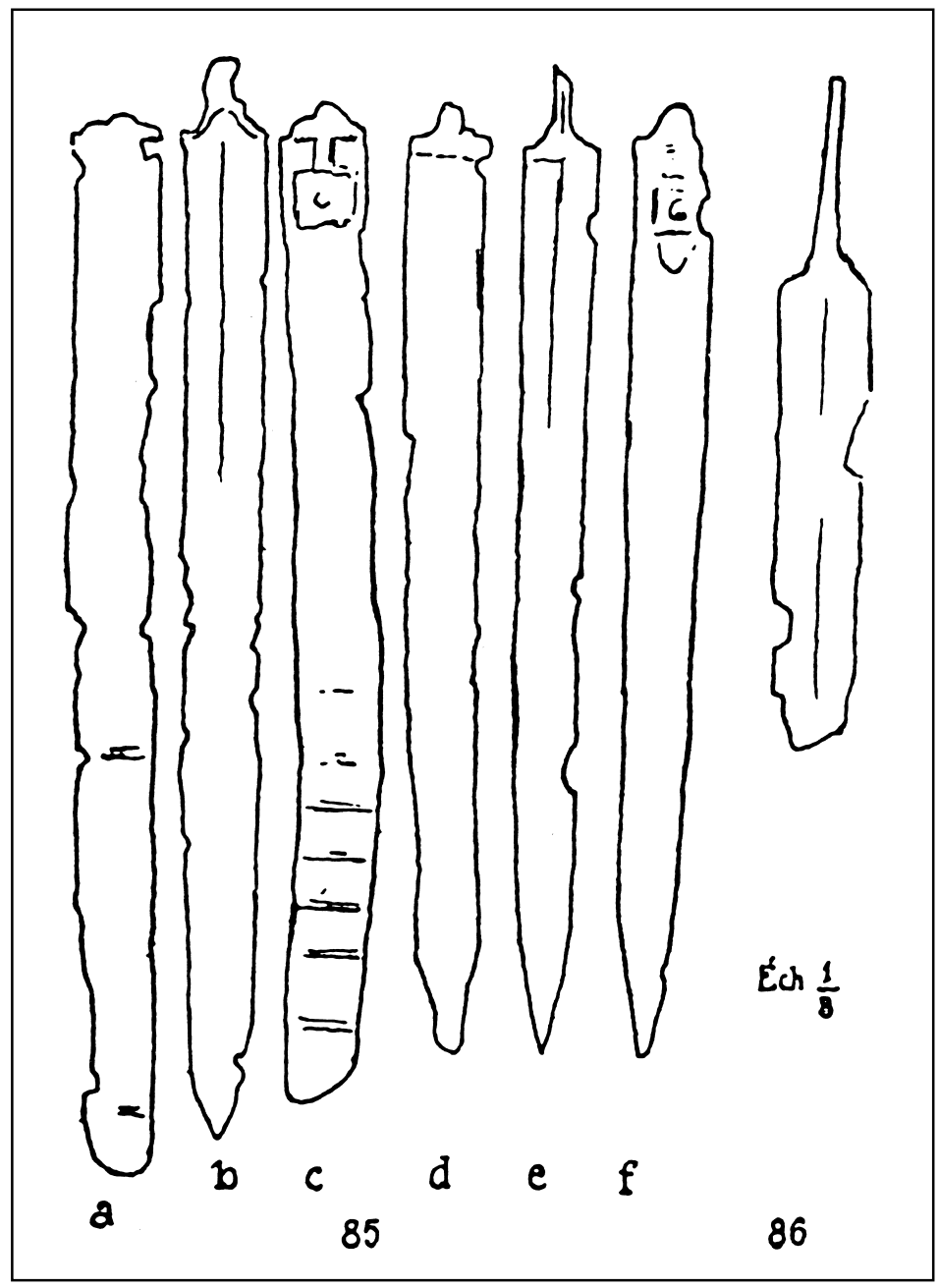

Fig. 1. Facsimilé des Figures 85 et 86 de l'ouvrage de COUISSIN (Couissin) 1926 illustrant son indentification du gladius hispaniensis au temps de César parmi les armes exhumés sur le site d'Alise-Sainte-Reine (Fig. 85 Epées d'Alesia, RA, 1864, pl. XXIII. Fig. 86 Epée d'Osuna, (Nouv. Arch. des miss, t. XIII, $4 .^{\mathrm{a}}$ fasc., pl. XXX)

Ce texte, laconique s'il en est, ne comporte de surcroît, aucune information morphologique précise. Cette identité «littéraire» du glaive romain républicain est pourtant sollicitée, depuis longtemps, bien au-delà de son contenu. Les fluctuations de son interprétation ne peuvent être réduites sans le recours à l'analyse exhaustive des rares documents disponibles.

Prise à la lettre, la définition de Polybe pourrait en effet s'appliquer sans difficulté à la plupart des épées gauloises contemporaines dont l'historien ignorait peut-être la morphologie précise et la diversité.

1.1.- Le port de l'épée «sur la cuisse droite» correspond précisément à une singularité des Celtes qui gagne rapidement l'ensemble de l'Europe laténienne à partir du $\mathrm{V}^{\mathrm{e}} \mathrm{s}$. Cette spécificité est liée à des options militaires privilégiant un style de combat très dynamique. Les deux constantes du fourreau laténien, d'une part, un étui métallique obtenu par le sertissage 


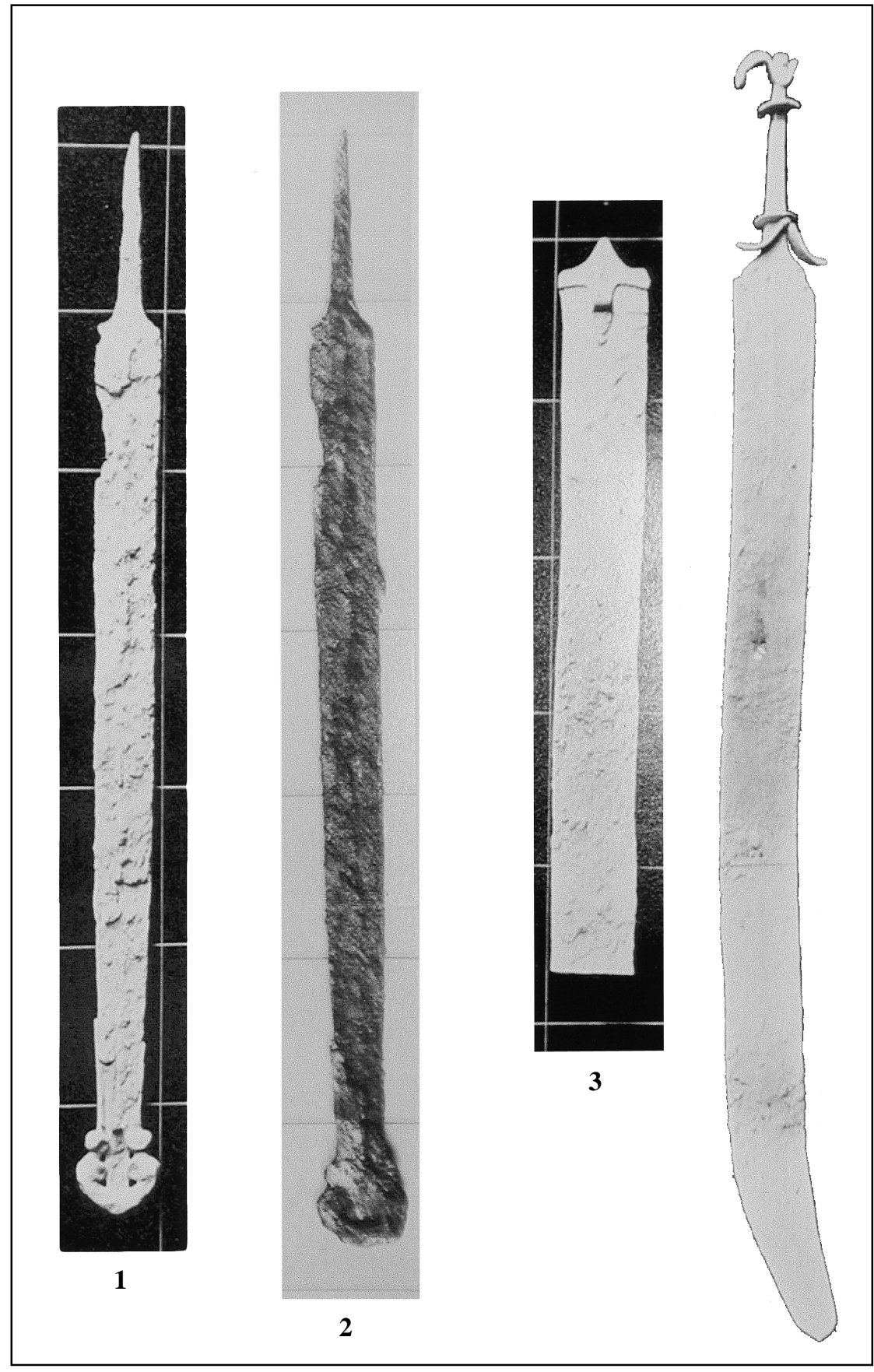

Pl. I. Deux épées gauloises «anachroniques» de la collection d'Alésia. Archives photographiques du Musée de Antiquités Nationales.

fig. 1- Epée de petit modèle, avec bouterolle du type dit Hatvan-Boldog, fin $\mathrm{IV}^{\mathrm{e}}$ début $\mathrm{III}^{\mathrm{e}}$ s. av. J.-C. (état originel).

fig. 2- La même épée dont la bouterolle a subi les transformations d'un «nettoyage» abusif.

fig. 3- Epée et fourreau dont la poignée et la pièce de suspension datent du dernier tiers du $\mathrm{III}^{\mathrm{e}}$ s. av. J.-C. 


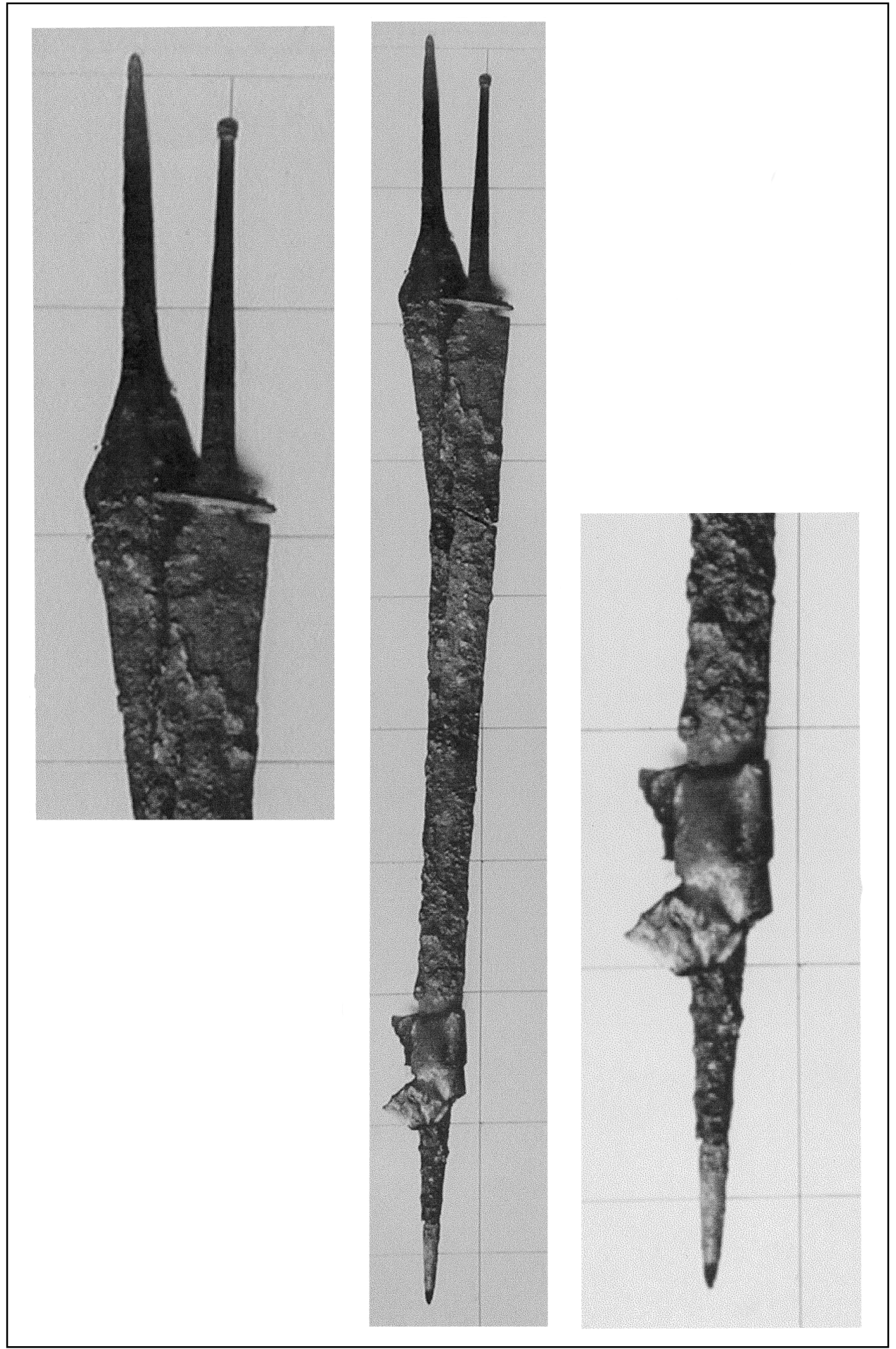

Pl. II. Deux épées de morphologie exogène dans la collection d'Alésia. Lames sans fourreau métallique soudées par la corrosion et ligaturées par une tôle en alliage cuivreux. (état originel. Archives photographiques du Musée des Antiquités Nationales). 
latéral de deux tôles minces, et d'autre part, la pièce de suspension verticale soudée sur l'arrière de l'étui, résultent de cette spécificité (Rapin 1991). L'ensemble épée-fourreau est solidaire d'un ceinturon adapté à la suspension de l'arme sur la hanche droite répondant aux contraintes imposées par la mobilité des combattants celtes.

A l'opposé, le mode de suspension de type grec sur le flanc gauche constitue une réponse au mode de combat plus statique des hoplites. Cette formule de suspension reste longtemps dominante dans les péninsules helléniques et italiques (Rapin 1991). Sa variante ibérique adaptée à d'autres styles de combat, est décalée sur l'avant du corps ${ }^{2}$. Utilisée pour la suspension de la falcata, elle viendra se superposer au mode de suspension des épées laténiennes adoptées par les Ibères et leurs voisins Celtibères. Leur adaptation à la suspension latérale par deux anneaux (ou parfois trois) est cependant différente du système à quatre anneaux utilisé en Grèce c'est-à-dire, celui qui sera exploité plus tard pour les gladii postérieurs à la Guerre des Gaules, soit un héritage plus hellénique qu'hispanique.

1.2.- La deuxième information de Polybe sur l'origine hispanique du gladius suscite depuis longtemps une abondante littérature dont témoigne le bilan effectué récemment par Fernando Quesada (Quesada 1997a, p. 262). Or, malgré l'importante documentation relative à l'armement protohistorique de la péninsule il faut bien reconnaître que la recherche d'une filiation directe entre une de ses armes de poing et les glaives romains connus, reste très décevante. Les ressemblances ponctuelles évoquées ça et là à propos des lames de poignard, leurs étuis ou leur suspension, participent d'un fonds traditionnel très ancien et commun à l'ensemble des armes méditerranéennes et même de l'Europe moyenne depuis le premier âge du Fer. En aucun cas ce type d'affinités très générales ne pouvait justifier le choix de ce qualificatif de la part de Polybe dont l'expérience dans le domaine de la guerre est connue. L'héritage grec du gladius, pour la suspension de l'étui comme pour la lame ou la poignée paraît bien plus convaincant.

Concernant un éventuel prototype espagnol d'épée longue la contradiction serait encore plus flagrante puisque les Ibères comme leurs voisins Celtibères ont déjà adopté, au temps de Polybe, l'arme laténienne et son fourreau métallique. Le sens du qualificatif hispaniensis est certainement à chercher ailleurs, probablement du côté de la fabrication des armes romaines par les métallurgistes ibères. Depuis le début du $\mathrm{II}^{\mathrm{e}} \mathrm{s}$. l'ensemble du Levant espagnol bascule dans l'orbite économique et politique de Rome. Une telle exploitation du savoir-faire local est tout à fait vraisemblable compte tenu de sa réputation de qualité. Cette opinion, déjà formulée par H. Sandars en 1913, offre plus de cohérence avec les données archéologiques que la quête du prototype espagnol du gladius (Quesada 1997a, p. 265).

1.3.- La «pointe remarquable» ne définit aucune forme précise. Cependant, le problème de la morphologie des «pointes» d'épée nécessite une mise au point indispensable.

En effet, un des clichés les plus récurrents depuis un siècle concerne la diachronie de l'épée celtique; courte et pointue au $\mathrm{V}^{\mathrm{e}}$ s. elle aurait évolué vers une forme de plus en plus longue à extrémité arrondie au $1^{\mathrm{er}}$ siècle av. J.-C. En réalité, les épées longues et pointues existent dès le début de La Tène au $\mathrm{V}^{\mathrm{e}}$ s. (cf. note 3). Leurs équivalentes sont toujours présentes quatre siècles plus tard (Pl. III, fig. 3 et 4). En outre armes longues et courtes, parfois même aux extrémités mousses ou aiguës, peuvent coexister en fonction des spécialisations de leurs propriétaires. Pendant la première moitié du II $^{\mathrm{e}} \mathrm{s}$. l'allongement spectaculaire de l'arme pour une nouvelle adaptation au combat à cheval produit deux effets pervers: l'un sur la morphologie de

2 Ce mode de suspension des armes de poing situées à l'horizontale dans le creux de la taille est clairement figuré sur les sculptures de guerriers du Cerillo Blanco à Porcuna (Andalousie). 
l'extrémité des épées de cavaliers, l'autre sur la bouterolle des fourreaux métalliques, en général (Rapin 1999).

— Le premier répond aux nécessités du développement des techniques du combat de taille à compter du $\mathrm{III}^{\mathrm{e}}$ s.: un sabre droit dont l'extrémité s'émousse peu à peu jusqu'à devenir demicirculaire (Pl. III, fig. 1). Les bouterolles adaptées à ce type de lame s'allongent et s'arrondissent et parfois même s'évasent pour aboutir à de nouvelles formules de fourreaux tels ceux de type Ormes et de Ludvigshafen à compter du milieu du $\mathrm{II}^{\mathrm{e}}$ s. (Schaaff 1984) (Rapin 1999, fig. 10 et 11) (Pl. IV, fig. 1 et 2).

Cette évolution conduit les armes de poing des phases LT.C2 et LT.D1 vers les lames les plus longues jamais fabriquées par les Celtes laténiens pour des cavaliers antérieurs d'au moins trois ou quatre générations à celles de leurs descendants d'Alésia (Rapin 1999). Certaines de ces lames dépassent les $95 \mathrm{~cm}$, ce qui correspond à un maximum, compte tenu de l'amplitude du geste nécessaire pour dégainer une telle arme (Pl. III, fig. 1 et 2).

- En second lieu, à l'intérieur de ces fourreaux aux bouterolles arrondies, les analyses radiographiques font apparaître toute une gamme de variations de pointes, triangulaires courtes, ogivales plus ou moins longues et parfois même très effilées. Ces bouterolles parmi les plus évasées dissimulant des pointes remarquables ont abusé des générations d'archéologues (Pl. III, fig. 3 et 4 et Pl. IV, fig. 1 et 2).

En outre, la tendance à l'allongement excessif de la lame tend à s'atténuer pendant le $1^{\mathrm{er}}$ siècle av. J.-C. Les armes gauloises contemporaines du siège d'Alésia de la planche V, montrent que pour certaines épées de fantassins ou sabres de cavaliers, les Celtes reviennent à des dimensions plus modestes, (entre 70 et $75 \mathrm{~cm}$ de longueur de lame). Leurs bouterolles rondes ou évasées gainent des extrémités de lames très diverses $(\mathrm{Pl} . \mathrm{V})$.

Ces variantes contemporaines peuvent correspondre, à l'instar des périodes précédentes, à des militaires aux spécialisations différentes. L'hypothèse de variantes régionales reste difficile à vérifier du fait de la rareté relative des sépultures contemporaines sur l'ensemble du domaine laténien pendant le dernier siècle de l'Indépendance.

En résumé, si l'extrémité mousse d'un sabre de cavalerie suffit à identifier une arme laténienne, la «pointe remarquable» (effilée?) d'une épée d'estoc ne constitue pas un critère déterminant pour différencier, à lui seul, les épées gauloises et romaines.

1.4.- Enfin, la dernière information livrée par Polybe, à propos de l'usage de taille des deux tranchants du gladius, correspond depuis l'âge du Bronze à la majorité des épées de l'Europe moyenne et méditerranéenne. L'historien, ex-militaire grec, souhaitait-il différencier le gladius hispaniensis de l'autre arme de poing des Ibères; la falcata? Or, tout comme ses équivalents grecs et italiques, kopis et machaïra, la falcata était de moins en moins d'actualité au temps de Polybe... (Quesada 1997a, p. 79-81). Cette fonction mixte de taille et d'estoc est de plus accompagnée de l'appréciation qualitative: «... parce que sa lame est forte et solide ...». La redondance des deux adjectifs dans un texte aussi bref n'est probablement qu'apparente: ... lame forte associée à l'usage de taille suggère peut-être un concept d'arme longue et puissante qui se distinguerait des glaives antérieurs dont la morphologie nous est inconnue... et solide, procède d'une appréciation qualitative qui nous renvoie à l'hypothèse de sa fabrication hispanique. Ce type d'appréciation subjective soulignerait, dans ce cas, les qualités de la métallurgie ibérique et les défaillances de son homologue romaine. Polybe est coutumier des appréciations subjectives aux connotations propagandistes. On sait, par exemple, que l'amalgame de sources concernant les deuxièmes Guerres puniques et la pratique du sacrifice des armes dans les sanctuaires gaulois est à l'origine du cliché du galate redressant avec le pied son épée ployée par le premier coup de taille. L'évocation de Tite-Live parlant des «terribles blessures» infligées par les glaives de l'armée de Paul-Emile à Pydna relève de ce même arrière plan idéologique. 


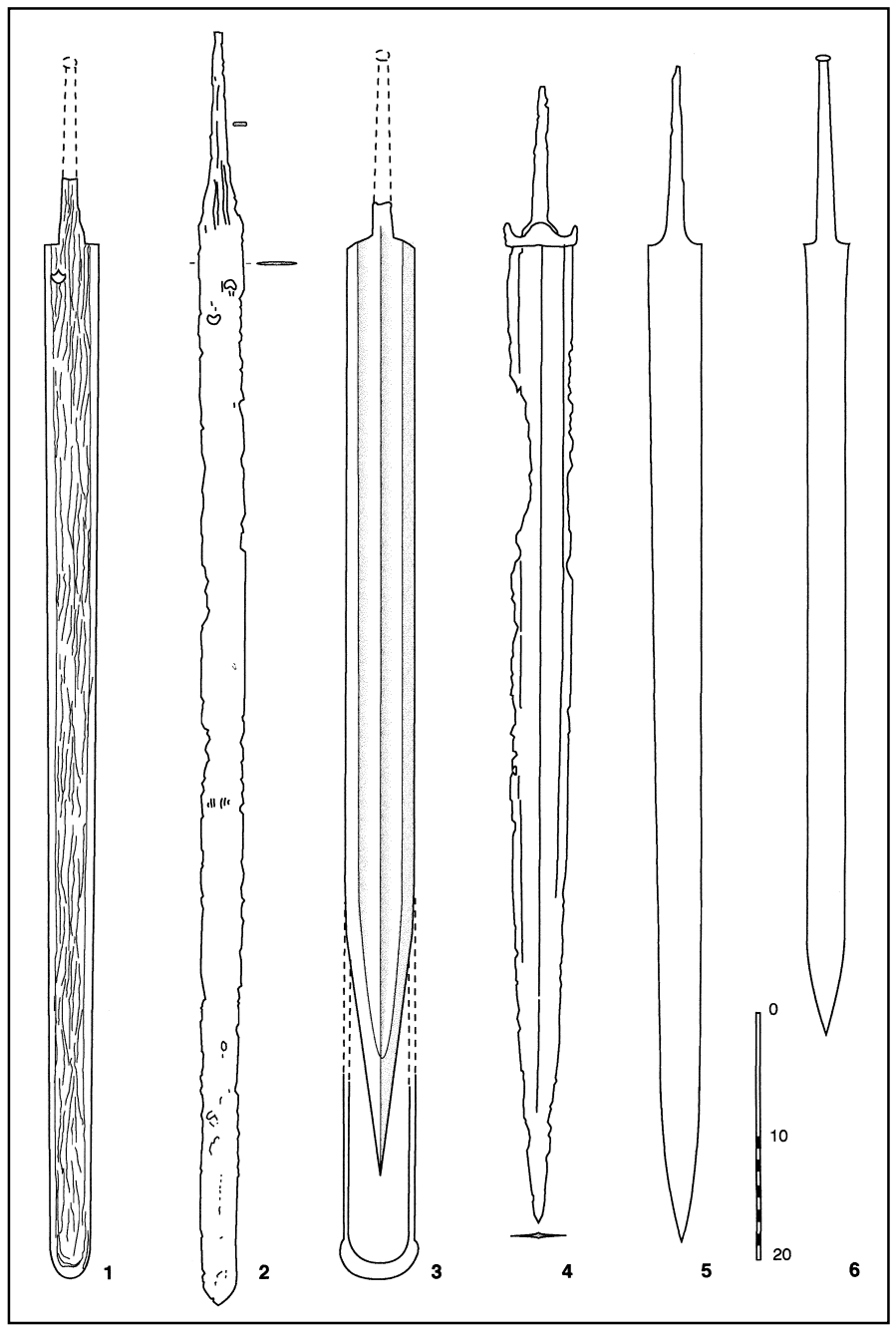

Pl. III. Quelques variations morphologiques des poignées et des extrémités d'épées gauloises et romaines.

fig. 1- Sabre de cavalerie laténien de Pont d'Arches (Seine-Inférieure). Epaulement anguleux de la soie, extrémité arrondie de la lame (milieu II ${ }^{\mathrm{e}}$ s. av. J.-C.). (inédit dessin A. Rapin).

fig. 2- Sabre de cavalerie laténien du Chalonnais (Musée Denon, Chalon-sur-Saône). Epaulement oblique de la soie, extrémité ogivale de la lame (milieu II ${ }^{\mathrm{e}}$ s. av. J.-C.). (d'aprés Bonnamour).

fig. 3- Epée laténienne à lame cannelée et extrémité très éffilée (dragage de l'Ain, Musée des Antiquités Nationales) $\left(2^{\text {ème }}\right.$ moitié du II s. av. J.-C.) (cf. Pl. IV, fig. 2). (inédit dessin A. Rapin).

fig. 4- Epée laténienne de même structure que la précédente draguée à Pouilly-sur-Saône (Côte-d'Or). Croisière de garde semblable à celle de l'épée $\mathrm{n}^{\circ} 1$ d'Alésia (cf. Pl. V). Musée Denon, Chalonsur-Saône ( $2^{\text {ème }}$ moitié du II ${ }^{\mathrm{e}}$ s. av. J.-C.). (d'aprés Bonnamour).

fig. 5- Schéma restituant la spatha précoce de Rottweil (d'après Feugère 1993) $\left(1^{\mathrm{er}}-\mathrm{II}^{\mathrm{ème}}\right.$ ap. J.-C. $)$.

fig. 6- Schéma restituant la spatha précoce de Pontoux (Doubs). Musée Denon, Chalon-sur-Saône, d'après Bonnamour ( $1^{\text {er }}$ s. ap. J.-C. $)$. 


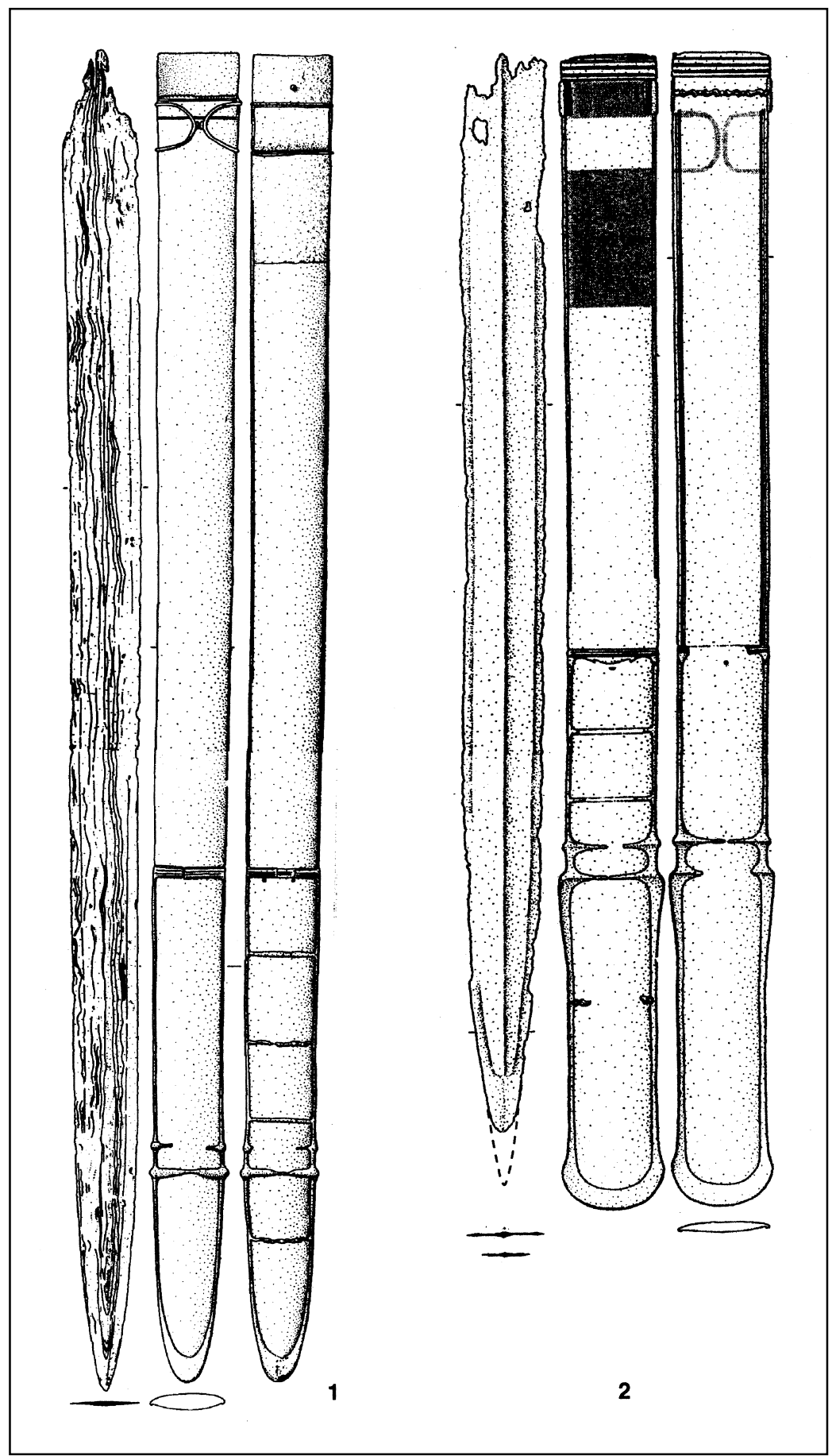

Pl. IV. Epées laténiennes pointues et leur fourreau en Al. cu, à bouterolle arrondie (type Ormes et type Ludvighaffen.

fig. 1- Ormes (Saône-et-Loire), Musée Denon, Chalon-sur-Saône, d'après Bonnamour (début $1^{\mathrm{er}}$ s. av. J.-C.).

fig. 2- La Saône à Pouilly (Côte-d'Or), Musée Denon, Châlonsur-Saône, d'après Bonnamour (fin $\mathrm{II}^{\mathrm{e}} \mathrm{s}$. av. J.-C.). 
En définitive quelles que soient les sources, écrites ou orales, anciennes ou récentes, utilisées par Polybe, sa définition du glaive romain est non seulement peu exploitable mais elle induit des problématiques sans issue dans l'état actuel de la documentation archéologique. Les recherches sur le qualificatif «hispaniensis» semblent prématurées faute de critères d'identification plus précis pour étoffer le corpus.

Si la corrosion explique l'essentiel des insuffisances dans l'observation des documents, les moyens pour la combattre; analyses radiographiques et technologiques, restaurations et nettoyages etc... ne m'ont pas semblé très sollicités jusqu'à présent. La grande majorité des armes du corpus est analysée à l'état brut de corrosion et, lorsque les gangues sont décapées, c'est le plus souvent sans les investigations radiographiques préalables indispensables.

\section{LES CRITÈRES MORPHO-TECHNOLOGIQUES ISSUS DES DOCUMENTS ARCHÉOLOGIQUES}

Dans la continuité des approches technologiques de l'Ecole anglaise et des recherches de son promoteur H.R. Robinson, P. Connolly prend d'emblai en compte deux critères-clé jamais évoqué par Polybe à savoir, une lame aux tranchants curvilinéaires associée aux matériaux organiques de son fourreau (Connolly, JRMES 8, 1997).

Si l'on souhaite différencier le glaive romain de ses homologues gaulois ou germanique c'est en effet par le profil légèrement sinueux de ses tranchants. P. Connolly désigne par le terme «waisted» l'étranglement de ce type de lames qui leur a valu le qualificatif de «pistiliforme» depuis le début de la métallurgie.

Ce vieil héritage de l'âge du Bronze et du premier âge du Fer européen est devenu peu à peu un archaïsme méditerranéen.

C'est en effet en Europe moyenne que l'on romp avec cette tradition vers la fin du premier âge du Fer, en particulier avec les nouvelles épées à tranchants parallèles de La Tène A. La mise au point de leur fourreau métallique, avec des gouttières de sertissages tubulaires et rectilignes, est fonctionnellement incompatible avec un profil de lame sinueux. La seule exception concerne certaines armes courtes des Celtes aux fonctions plus symboliques que militaires. Ces poignards dits «anthropoïdes» conserveront quelques temps ce profil archaïque avec, incrustés sur leur lame, les symboles astraux du soleil et de la lune.

En revanche, pendant cinq siècles, les épées des Celtes ne dérogeront jamais à ces trois principes: fourreau métallique, avec pièce de suspension au revers et rectitude des tranchants parallèles. Les Romains n'adopteront que très tardivement, sous l'Empire, certaines de ces options, pour le gladius comme pour la spatha. En revanche ils resteront fidèles au fourreau de bois pour l'épée, parfois enrobé d'un étui métallique rudimentaire sur leur poignard.

A partir de ce constat très simple une grande partie de la documentation exploitée lors de la réunion de Montpellier (ROMEC 1996) devient caduque.

Un des trois exemplaires de Šmihel sélectionnés par Horvat (Horvat JRMES 8, 1997) est une épée laténienne du $\mathrm{V}^{\mathrm{e}}$ s. av. J.-C. ${ }^{3}$. Celui de Dodone (JRMES 8, p. 99, fig. 13), la série du dépôt de La Azucarera (JRMES 8, p. 241-245) comme celles de La Osera, de Quintana de Gormas, du Cigarralejo (JRMES 8, p. 258-260) ou encore de Turo dels dos Pins en Catalogne (JRMES 8, p. 263), de Herdade das Cases au Portugal (JRMES. 8 p. 132) et d'autres encore, sont aussi des épées de type laténien. Le fait que leur système de suspension soit quelquefois adapté aux comportements des militaires locaux, voire même lorsqu'elles sont gainées dans un fourreau en bois ne change rien à la filiation laténienne de l'arme et de son utilisation.

3 Cette épée figurée à côté des deux glaives de Šmihel (Horvat 1997, p. 114) présente non seulement toutes les caractéristiques d'une arme laténienne mais les divers indices technologiques et morphologiques visibles (section de la soie, épaulement, largeur de la lame, forme de la pointe et cannelures...) relèvent des traditions techniques du $\mathrm{V}^{\mathrm{e}} \mathrm{s}$. av. J.-C. Il s'agit probablement d'une des épées laténiennes les plus anciennes de cette région des Balkans. 
Le deuxième critère d'identification, à savoir, le fourreau de bois et son système de suspension approprié, correspond également aux mêmes archaïsmes et aux mêmes héritages que celui des lames. Grecs, Italiques et même Ibères, seront eux aussi longtemps fidèles à ce type d'étui et à sa suspension. Cependant les matériaux organiques du fourreau plus ou moins minéralisés et mêlés aux produits de corrosion, induisent des difficultés pour la lecture des lames masquées par les gangues superficielles.

Plusieurs cas de figures perturbent les identifications. Un fourreau en matériaux organiques ne peut être automatiquement induit par la seule absence de l'étui métallique. Quantité d'épées laténiennes exhumées depuis le $\mathrm{XIX}^{\mathrm{e}} \mathrm{s}$. ont perdu leur fourreau originel en fer, parfois dès leur prélèvement en sépulture mais surtout, dans les réserves des musées où l'érosion continue son oeuvre destructrice.

En second lieu, des matériaux organiques (cuir, bois, tissus) peuvent se minéraliser fréquemment par dessus les tôles de l'étui métallique au point d'entraîner des identifications farfelues telles celles de fourreaux en «tissu», en «peau de bête» ou en «bois» dont on a pu démontrer l'inexactitude ${ }^{4}$.

Enfin, lorsque les produits de corrosion piègent les composantes organiques d'un fourreau un cliché radiographique bien ciblé permet de révéler avec précision la morphologie des tranchants de la lame sous-jacente, comme sa structure nervurée, cannelée, lenticulaire etc...

Quelle est la forme exacte des lames enfouies dans les fourreaux de Delos, d'Es Soumâa, d'Osuna5. Le gain de précision apporté par les clichés RX sur les armes de Giubiasco, Šmihel, Azucarera mais aussi sur celles de Turo dels dos Pins, Murcia, El Cigarralejo ou Mouriès et bien d'autres serait à même de changer la nature des données, des chronologies et des problématiques. A l'inverse, l'épée de La Caridad (Teruel) (JRMES 1997 8, p. 188, fig. 33) est qualifiée de type La Tène alors que les indices visibles avant toute analyse radiographique devraient permettre de l'intégrer dans le corpus des glaives républicains (cf. chapitre 4, 2, Pl. IX, fig. 3).

Loin d'avoir des conséquences négatives en éliminant éventuellement quelques exemplaires d'un corpus déjà bien maigre d'épées romaines, les travaux de restaurations précédés des investigations indispensables, tendent plutôt à accroître la quantité et la qualité des armes exploitables pour les périodes antérieures, contemporaines, ou postérieures aux guerres césariennes ${ }^{6}$.

\footnotetext{
4 Un exemple récent de ce type d'identification hâtive d'un étui en peau de bête vient d'une sépulture de guerrier celtique inhumé sur une peau de bovin dont la fourrure minéralisée avait recouvert le fourreau en fer. cf. Lourdeaux Sylvie; La sépulture celtique de Leletton (Vendée). Colloque AFEAF de Nantes 1999 (à paraître dans la Revue Archéologique de l'Ouest).

5 Sous les gangues des corrosions une série de clichés RX de l'épée d'Osuna vient d'être réalisée au Musée des Antiquités Nationales. Elles mettent en évidence le profil légèrement pistiliforme de la lame qui devient de ce fait un gladius vraisemblable. (Remerciements à Françoise Douhaut du M.A.N.).

6 Un nombre relativement important de glaives inédits mal identifiés et dispersés dans les réserves des musées pourrait bénéficier de cette investigation minimale par clichés RX. La variabilité de l'emplacement du «Waisted» plus ou moins décalé vers le haut ou la pointe de la lame pourrait par exemple intégrer la grille des critères diachroniques déterminants.
} 


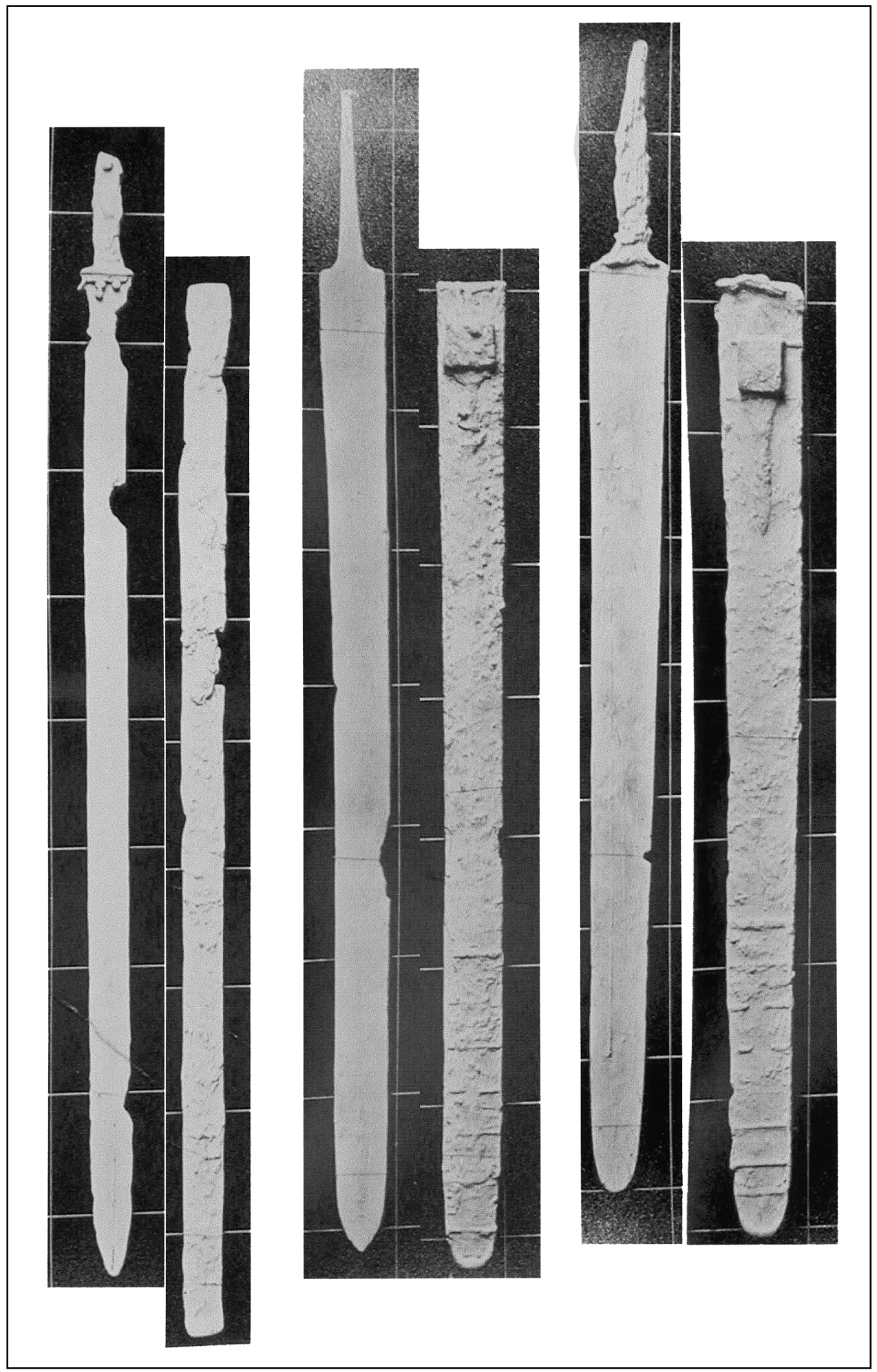

Pl. V. Trois épées gauloises d'Alésia, contemporaines du siège, les fourreaux et les lames ont été demontées au XIX ${ }^{\mathrm{e}}$ s. (Archives photographiques du M.A.N.). 


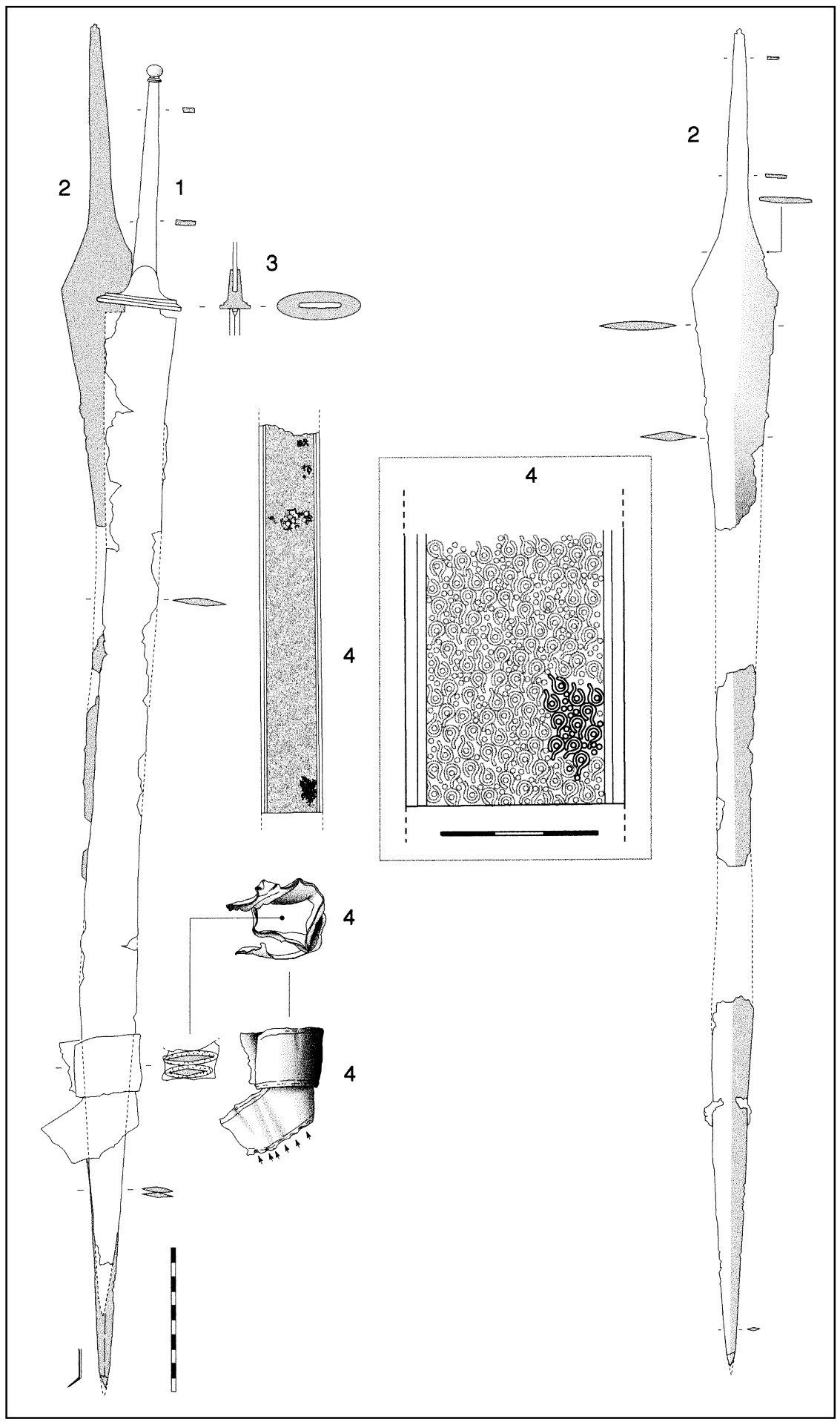

Pl. VI. fig. 1 et 2.- Les deux épées d'Alise superposées et «ligaturées». fig. 3- Vues de profil et de dessous de la croisière de l'épée $\mathrm{n}^{\circ} 1$. fig. 4- Différentes vues du fragment de tôle de fourreau en Al. cu avec développé et décor de chagrinage. 


\section{Les Glaives D’ALÉSiA ( $\mathrm{N}^{\circ}$ INVENTAiRE DU M.A.N. 24 337) (Pl. II, VI, VII, VIII)}

Les clichés effectués pendant les années 1860 montrent l'état de l'ensemble des trois objets associés lors de leur arrivée au Musée des Antiquités Nationales (Pl. II) (cliché 870, boîte 77; page 277 de l'album).

Les deux épées superposées sont partiellement soudées par la corrosion du fait de la pression exercée par l'enroulement d'un fragment de fourreau laténien en bronze. Cette association relève d'un acte délibéré dont l'intérêt va au-delà des analyses typo-chronologiques (cf. ci-après chap. 6).

\section{1- Epée $n^{\circ} 1$ (Pl. VI, fig. 1 et Pl. VII, fig. 1)}

La plus courte et la plus complète des deux armes possède une lame «pistiliforme» de section lenticulaire, à pointe effilée, de $70 \mathrm{~cm}$ de long et $5 \mathrm{~cm}$ de largeur maximum. La soie se termine par un bouton en alliage cuivreux pour la fixation du pommeau. Une croisière en fer bien conservée termine du côté de la garde une poignée longue de 17,5 cm. Compte tenu de la constante ergonomique représentée par le poing fermé sur la fusée (entre 75 et $80 \mathrm{~mm}$ ) la longueur de cette soie suppose une garde et un pommeau volumineux.

Un phénomène similaire d'allongement des soies caractérise aussi les épées laténiennes à compter du $\mathrm{II}^{\mathrm{e}}$ s. av. J.-C. Lorsqu'elles atteignent 16 à $18 \mathrm{~cm}$ de longueur la présence d'un pommeau ovoïde ou sphérique de 5 à $6 \mathrm{~cm}$ de diamètre peut être induite. Une pièce de renfort de pommeau encore en place sur une épée de La Tène atteste cette morphologie (Vouga 1923) (Pl. X, fig. 1). Quant aux gardes métalliques à base rectiligne elles sont présentes également sur des épées gauloises aux tranchants parallèles telles celles de Saint-Laurent-des Arbres (Gard) (Barruol, Sauzade 1969) ou encore de Pouilly-sur-Saône (Côte-d'Or) (Pl. III, fig. 4 et Pl. X, fig. 2).

3.2- L'épée $n^{\circ} 2$ a connu en revanche quelques dégradations supplémentaires contemporaines ou postérieures à son dépôt au Musée des Antiquités Nationales qui ont conduit à une réparation de fortune à l'aide d'un pansement de plâtre entoilé. Mais cette intervention ancienne n'a pas été très appropriée pour sa protection à long terme et sa fragmentation s'est poursuivie entraînant la pulvérisation de certains tronçons de la lame. Fort heureusement, de part et d'autre de la «ligature», la tôle de bronze a induit une pression suffisante pour provoquer une adhérence des lames par la corrosion et conserver leur position relative initiale ce qui permet de restituer les dimensions de l'épée $n^{\circ} 2$. Sa lame originelle plus effilée que la précédente, mesurait entre 76 et $77 \mathrm{~cm}$ de longueur si l'on prend en compte la topographie initiale des deux deux lames enregistrée au XIX ${ }^{\mathrm{e}}$ s. (Pl. II). La soie qui a perdu son rivet terminal, mesure actuellement $18,5 \mathrm{~cm}$ de longueur, ce qui suppose une garde et un pommeau encore plus massifs que ceux de l'épée ${ }^{\circ} 1$. L'évasement triangulaire de la base de la soie induit une hauteur de 5 à $6 \mathrm{~cm}$ pour la garde (Pl. VI, fig. 2; Pl. VII, fig. 2).

Bien que nettement plus large que la précédente à son épaulement $(60 \mathrm{~mm}$ contre 50 $\mathrm{mm}$ ) sa lame se rétrécit rapidement (jusqu'à $24 \mathrm{~mm}$ ) pour se terminer, telle une rapière, par une longue pointe effilée. Sa fonction d'estoc est renforcée par l'arrête médiane (section losangée) qui commence à une quinzaine de centimètres sous la garde.

Enfin, pour terminer ce descriptif sommaire il est nécessaire d'insister sur la caractéristique commune des tranchants non parallèles des deux lames qui leur donne cette morphologie exotique évoquée plus haut. L'étranglement de leur profil respectif (Waisted), nettement plus prononcé sur l'épée $\mathrm{n}^{\circ} 2$, leur confère cet aspect «méditerranéen» dont les parallèles précis et bien datés sont encore rares. 


\section{ETUDES COMPARATIVES ET CHRONOLOGIES}

L'origine précise de la découverte de ces deux armes n'est pas connue et sa provenance globale d'Alise-Sainte-Reine ne peut que lui conférer un terminus ante quem approximatif, au plus tard, contemporain du siège. De plus les exemples de la planche I montrent que la provenance de certaines épées enregistrées en 1860 correspond plus probablement à celle d'un cimetière local peut-être mis au jour fortuitement par les fouilles des terrassiers césariens.

\section{1- Epée $n^{\circ} 1$ (Pl. VIII, fig. 1)}

Des deux armes c'est celle qui trouve les correspondants les plus proches dans l'arc alpin.

Ses dimensions, la section lenticulaire de sa lame, le raccordement de la soie à base plate par deux brefs épaulements rectangulaires et sa pointe triangulaire la rapprochent des deux glaives du cimetière tessinois de Giubiasco (Suisse) utilisés comme références par P. Connolly (JRMES 8, fig. 8, p. 51). Un supplément d'analyses radiographiques et un nettoyage des gangues à l'emplacement de la garde devraient mettre en évidence ce que l'on devine d'une pièce métallique campaniforme, proche de celle d'Alésia, réalisée en alliage cuivreux, au moins en surface, d'après Margarita Primas (Primas 1992), (Voir clichés RX em en annexe).

Cette formule d'épaulement anguleux a des équivalents sur des armes celtiques à compter de la transition LT.C2-LT.D1 soit dans le tiers central du II ${ }^{\mathrm{e}}$ s. av. J.-C. (Pl. III, fig. 1).

En revanche, concernant la chronologie de la tombe 119 de Giubiasco je ne partage pas le pessimisme de P. Connolly qui la juge impossible. La présence dans la panoplie d'un umbo de bouclier celtique à longues ailettes rectangulaires constitue au contraire un excellent repère temporel (JRMES, op. cit. p. 50). Parmi les 220 exemplaires d'umbos de boucliers analysés pour l'étude de l'armement du sanctuaire de Gournay-sur-Aronde (Oise), la série la plus importante est constituée d'une quarantaine de spécimens du même type et de mêmes dimensions que l'umbo de la tombe 119 de Giubiasco (Rapin 1988). De plus, cet umbo m'a déjà servi de jalon avec ses semblables de Slovénie, de Celje à Dobova, jusqu'en Hongrie à Pusztagéc (Rapin 1988, p. 71).

L'arme caractériserait un équipement de cavalier de la phase LT.C2 soit en gros la première moitié du $\mathrm{II}^{\mathrm{e}} \mathrm{s}$. av. J.-C. Le fer de lance de la tombe 119 de Giubiasco tout comme le crochet du ceinturon laténien sont également caractéristiques de ces panoplies. La forme plus fermée de la bosse centrale de l'umbo de Giubiasco, proche de la calotte sphérique, représente un indice d'évolution qui situerait l'ensemble de Giubiasco dans le tiers central du $\mathrm{II}^{\mathrm{e}} \mathrm{s}$. av. J.-C. Le casque de type Negau évolué, avec son rebord épais à deux cannelures, reste cohérent avec cet ensemble homogène, même si les datations récentes de son utilisation sont globalement légèrement postérieures (Egg 2000).

Pour autant, une éventuelle apparition de cette forme de gladius au $\mathrm{II}^{\mathrm{e}} \mathrm{s}$. ne présume en rien de la stabilité du type pendant un siècle. Elle constitue tout au plus un terminus post quem provisoire pour l'épée $\mathrm{n}^{\circ} 1 \mathrm{~d}$ 'Alésia qui pourrait être ainsi une arme contemporaine de la rédaction de Polybe.

\section{2- Epée $\mathrm{n}^{\circ} 2$ (Pl. IX, fig. 1)}

L'arme n'a pour l'instant que très peu de parallèles, le plus souvent sans contexte. Le large évasement triangulaire de la soie conçu pour une garde très massive se retrouve sur un gladius de Slovenie occidentale à Vrhnika (Horvat 1990). Avec une largeur de $56 \mathrm{~mm}$ à l'épaulement, ce gladius du Norique présente d'autres affinités avec celui d'Alésia (Pl. IX, fig. 3).

- Sa lame de $66 \mathrm{~cm}$ de longueur est certes moins longue et moins étroite mais sa morphologie et ses dimensions ont fait douter Jana Horvat de son appartenance à la famille gladius (Horvat 1990, p. 239). 


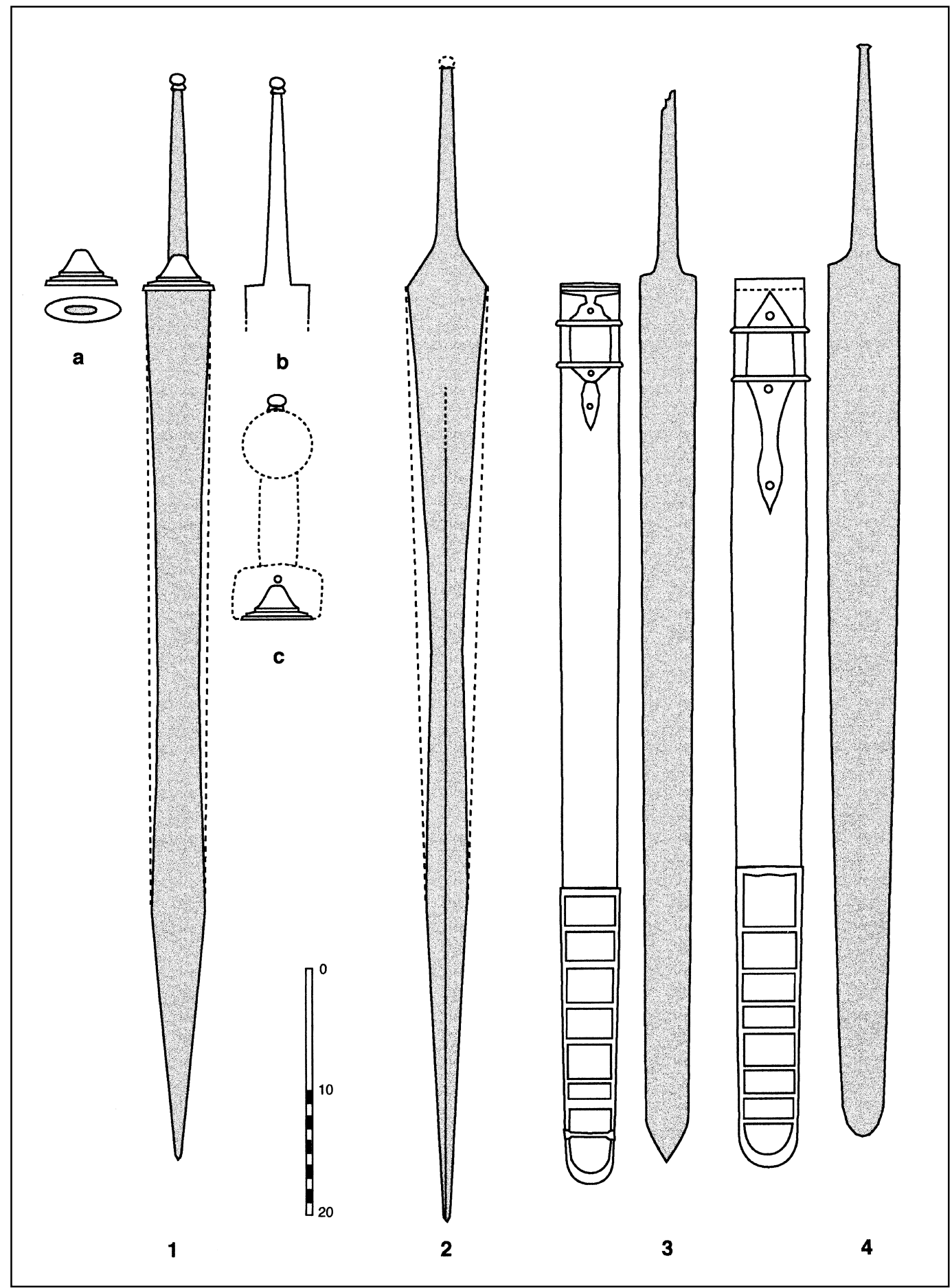

Pl. VII. Mise en évidence des différences de conception des lames romaines et gauloises.

fig. 1 et 2- Restitution schématique des deux glaives d'Alise (a-b-c: poignée et épaulement du $\left.\mathrm{n}^{\circ} 1\right)$.

fig. 3 et 4- Deux des épées gauloises d'Alise figurées dans la Pl. V avec leur extrémité triangulaire ou arrondie et leurs bouterolles identiques. 


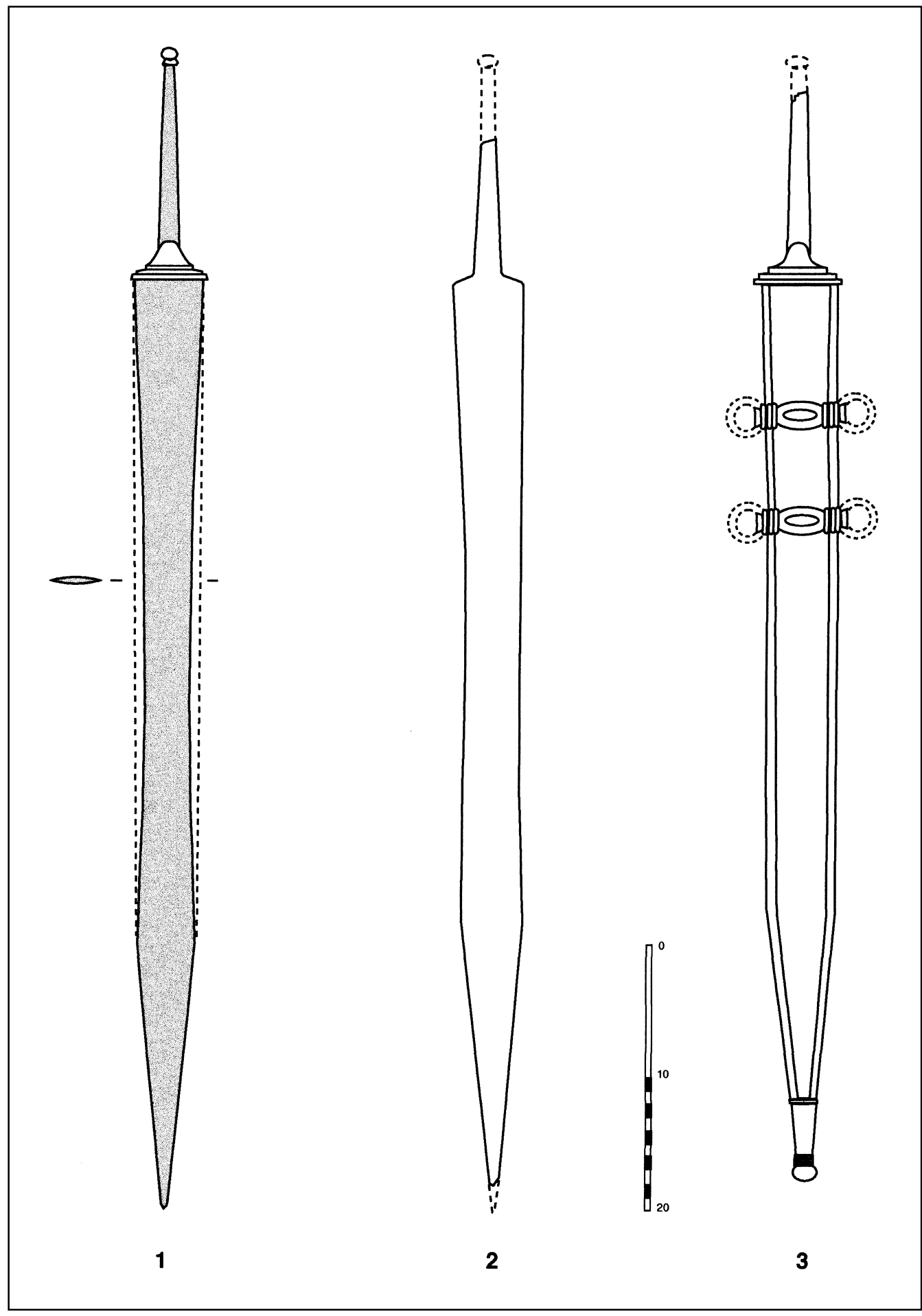

Pl. VIII. Le gladius $n^{\circ} 1$ d'Alise et ses parallèles de Giubasco: 1, Alise; 2, Giubiasco, tombe 471; 3, Giubiasco, tombe 119 avec restitution schématique du fourreau, de sa suspension et de la garde. (Restitution proposeeé avant la prise des clichés RX par le museé national de Zurich. Voir planche hors-texte p. 54). 
— Comme pour l'épée $\mathrm{n}^{\circ} 2$ d'Alésia sa section d'abord lenticulaire se transforme en section losangée.

- De même, sa pointe effilée est précédée d'un renflement bien marqué.

- La restitution de la soie lui confère une longueur totale initiale de $84 \mathrm{~cm}$ soit, un encombrement important proche de celui des armes d'Alésia.

Une lame pistiliforme très étroite malheureusement incomplète et beaucoup plus courte figure dans l'inventaire des épées d'Alise (Verchère de Reffye, op. cit, Pl. XXII). Parmi les armes de type laténien de Castille, Schüle fait figurer une autre longue rapière de ce type provenant de la tombe B d'Arcóbriga (Schüle 1969). Malheureusement son relevé schématique est trop sommaire pour exploiter la comparaison.

Enfin, l'épée de La Caridad citée en introduction possède quelques indices morphologiques communs avec l'épée $\mathrm{n}^{\circ} 2$ (cf. Supra. Chap. 2) (Pl. IX, fig. 2). L'évasement triangulaire de la soie, son «waisted» bien visible malgré la corrosion et sa longueur de lame restituable autour de $70 \mathrm{~cm}$, en font probablement un gladius républicain non encore répertorié (J.D. Vicente et al., JRMES 8, p. 188).

Les larges évasements triangulaires de la base de la soie ont une fois de plus des équivalents laténiens. Certaines des longues lames celtiques du milieu du $\mathrm{II}^{\mathrm{e}}$ s. sont en effet équipées de ce type de garde volumineuse assujetie sur une base très élargie de la soie (Pl. III, fig. 2).

Ainsi, malgré la différence marquée de leur conception, épées gauloises et romaines sembleraient suivre des évolutions parallèles. Les contacts de proximité, peuvent expliquer des «influences» réciproques. De plus certains recoupements d'innovations techniques de détail dont les initiateurs sont pour l'instant insaisissables, semblent naturels pour des militaires alternativement adversaires et alliés du fait de la pratique intense du mercenariat.

\section{GLADIUS OU SPATHA? (PL. X)}

La longueur totale de l'épée $\mathrm{n}^{\circ} 1$ est actuellement de $84 \mathrm{~cm}$. La restitution de la pointe cassée ajoute $3,5 \mathrm{~cm}$ soit $87,5 \mathrm{~cm}$. L'addition de l'extrémité de la bouterolle d'un fourreau du type de celui de Giubiasco permet d'estimer son encombrement originel autour de $90 \mathrm{~cm}$.

Pour l'épée $\mathrm{n}^{\circ} 2$ les mêmes calculs effectués à partir d'une lame de $76 \mathrm{~cm}$ auxquels s'ajoute une soie de $18,5 \mathrm{~cm}$ sans son bouton terminal conduisent à une estimation de longueur maximum comprise entre 98 et $99 \mathrm{~cm}$.

Un calcul de restitution semblable permet d'estimer les dimensions initiales des soies cassées des deux glaives de Giubasco. Compte tenu des constantes imposées par l'ergonomie de la poignée, les deux glaives du Tessin atteindraient une longueur totale de $86 \mathrm{~cm}$ pour celle de la tombe 119 et $90 \mathrm{~cm}$ pour l'arme de la tombe 471 .

L'arme de Vrhnika devait atteindre également $86 \mathrm{~cm}$ avec son fourreau et celle de $\mathrm{La}$ Caridad près de $90 \mathrm{~cm}$.

Dans cet ordre de dimensions restituées et donc comparables les épées d'Alésia comme leurs homologues méridionales se situent bien au delà du maximum des tableaux dimensionnels comparatifs proposés par M. Feugère (Feugère 1993, p. 140) ou par F. Quesada (Quesada 1997b, p. 256). De telles longueurs ne seront atteintes plus tard que par les spathae impériales.

Seule la spatha dite précoce de Rottweil (Feugère 1993, p. 147) dépasse de $3 \mathrm{~cm}$ l'épée $\mathrm{n}^{\circ} 2$ d'Alésia (Pl. III, fig. 5). Ces grandes lames impériales aux tranchants rectilignes peuvent, par ailleurs, prêter à confusion. Retrouvé en dehors de tout contexte le sabre aux tranchants parallèles de Rottweil pourrait fort bien être assimilé à ses équivalents laténiens des $\mathrm{II}^{\mathrm{e}}$ et $1^{\mathrm{er}}$ s. av. J.-C. comme aux épées du Haut Moyen-Age, plus tardives d'au moins sept siècles (Pl. III, fig. 4). Quelques critères morphologiques anciens sont parfois perceptibles sur certaines spathae, bien conservées, telle celle de Pontoux (Bonnamour, 1990) (Pl. III, fig. 6). Le 
renforcement rhomboïdal de sa pointe, la légère convergence des tranchants inscrivent cette spatha romaine précoce dans la lignée des glaives. Cependant ce sont les fourreaux de bois et cuir et leurs accessoires métalliques qui permettent la distinction immédiate entre des armes laténiennes, romaines et médiévales, très proches par leur fonction.

En effet, si l'on prend en considération l'encombrement de ces épées en regard de la taille moyenne d'un individu il est clair que leur attribution aux cavaliers semble la plus logique (Rapin 1991). Cette corrélation est parfois confirmée par l'archéologie. Ainsi l'aristocratie militaire du pays Trévire, contemporaine de César, signale cette spécialisation militaire par la présence d'éperons accompagnant l'épée longue gauloise dans le mobilier funéraire (Metzler $e t$ al. 1991). Or, il se trouve que dans une de ces sépultures celtiques aristocratiques c'est un long gladius qui remplace l'épée gauloise: tombe C de Goeblange-Nospelt (Luxembourg) (Metzler, op. cit, fig. 86, p. 113).

L'iconographie impériale des stèles funéraires exploite fréquemment l'ancien topos du cavalier terrassant le barbare. Le pommeau sphérique ou bulbeux de leur grande spatha se situe au contact de la poitrine. L'importance signalétique de ces grandes poignées se traduit souvent par une tendance à l'hypertrophie et des disproportions flagrantes (Pl. X, fig. 4).

Selon toute vraisemblance les deux épées d'Alésia pourraient être aussi des armes d'une classe équestre républicaine. Après les deuxièmes guerres puniques et les défaillances de la cavalerie romaine, Rome a dû faire appel aux troupes spécialisées de ses adversaires habilement exploitées par Hannibal. Les cavaleries légères des Numides et lourdes des Celtes et des Ibères ont dû intégrer plus massivement les armées romaines à compter du $\mathrm{II}^{\mathrm{e}} \mathrm{s}$. pour en devenir la composante exclusive avec les réformes de Marius à l'articulation du $1^{\mathrm{er}} \mathrm{s}$. av. J.-C. Les équipements mixtes du type de celui de la tombe 119 de Giubiasco témoigneraient de telles intégrations de troupes mercenaires d'origine celtique dès le $\mathrm{II}^{\mathrm{e}}$ s. av. J.-C. Peut-être spatha avant la lettre, ce module de gladius à longue lame de taille et d'estoc et fourreau de bois constituerait avec le casque leurs seuls indices d'une acculturation militaire italique.

A l'opposé, les troupes montées gauloises, intégrées dans l'armée romaine ou enrôlées de force par César, conservent-elles toujours leur équipement et leur mode de combat habituel ? César ne le dit pas clairement mais cela est probable.

Même si l'élaboration de la statue de Vachères est plus tardive, son épée dont la garde est très proche de celle d'Alise, est probablement une arme de cavalier. Cette épée que j'ai longtemps considérée comme laténienne, avant la restauration des armes d'Alésia, m'apparait de plus en plus comme un possible gladius ou une spatha précoce témoin de cette interpénétration continue entre les équipements militaires du Nord et du Sud (Pl. X, fig. 3). La poursuite du processus d'intégration des cavaleries celtiques, ibériques ou germaniques sous l'Empire est visible sur la stèle de C. Romanius Capito (Chew 1991). Cet officier de cavalerie originaire de Celje en Norique, près de Vhrnika, mort au premier siècle de notre ère s'inscrirait dans la suite logique de cette tradition très ancienne.

\section{LA TÔLE DE FOURREAU EN ALLIAGE CUIVREUX (PL. VI, FIG. 4)}

Cette tôle de $270 \mathrm{~mm}$ de longueur correspond à un fragment découpé de la plaque de droit d'un fourreau laténien de $42 \mathrm{~mm}$ de large. Ses flancs sont en effet rigoureusement parallèles et sa largeur maximum insuffisante pour gainer l'une ou l'autre des deux lames. La tôle relativement épaisse $\left(5 / 10^{\text {ème }}\right.$ de $\left.\mathrm{mm}\right)$ porte les traces d'un décor de type «chagrinage» délimité par un double bandeau longitudinal de lignes gravées parallèles aux flancs de la plaque. Ce type d'ornement très prisé des Celtes est ici obtenu par la répétition d'une estampille en forme de point d'interrogation ou demi-esse, agrémenté de petits cercles disposés au centre et à la périphérie du motif (Pl. VI, fig. 4). L'exploitation du signe demi-esse devient récurrente soit sous forme de grandes images ou d'estampilles à compter de la fin du $\mathrm{III}^{\mathrm{e}} \mathrm{s}$. av. J.-C. 


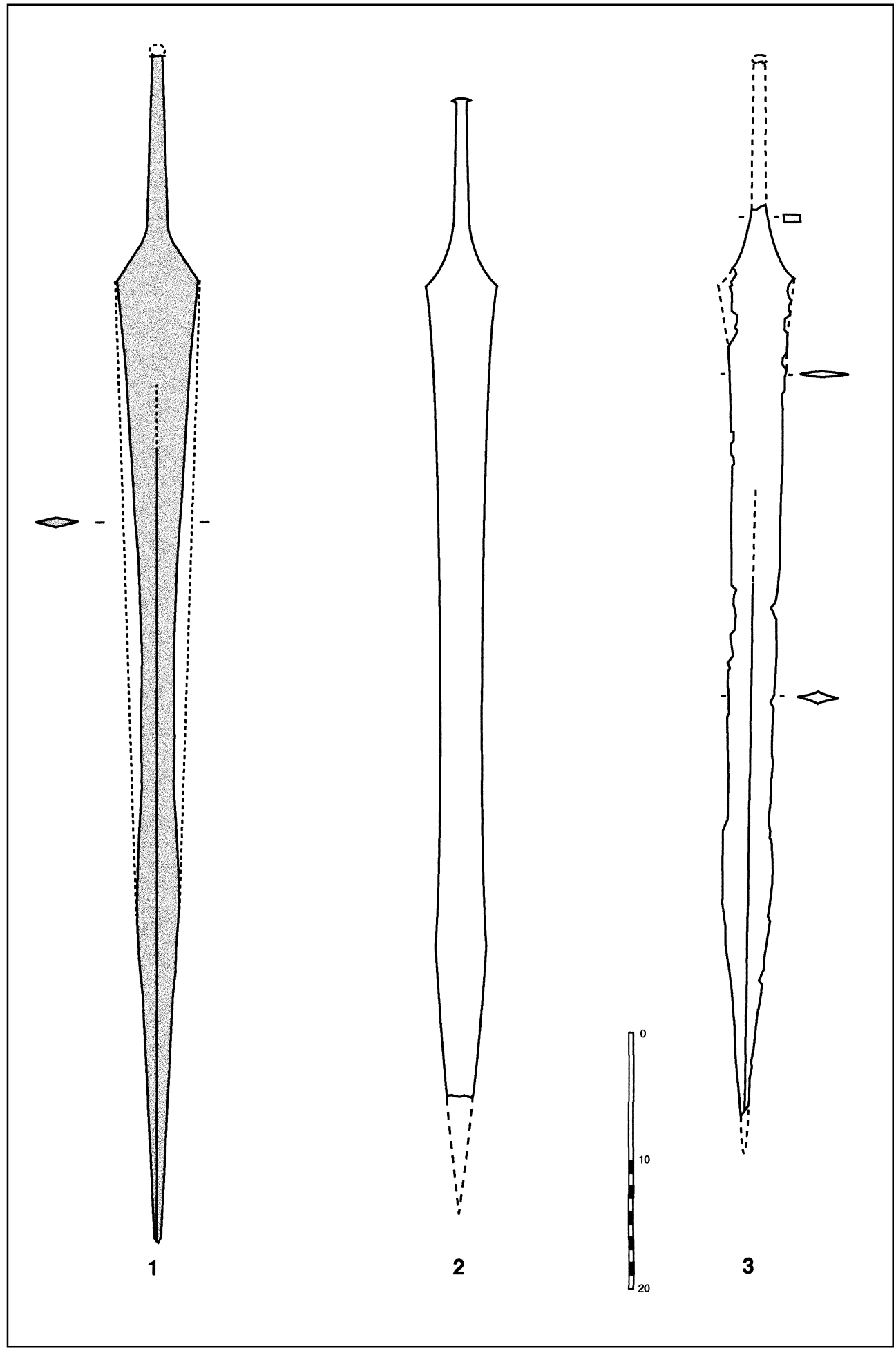

Pl. IX. Le gladius n ${ }^{\circ} 2$ d'Alise et ses parallèles: 1, Alise; 2, La Caridad (Espagne), restitution hypothétique d'après photographie avant radiographie et nettoyage; 3 , Vrhnika (Slovénie) d'après J. Horvat. 


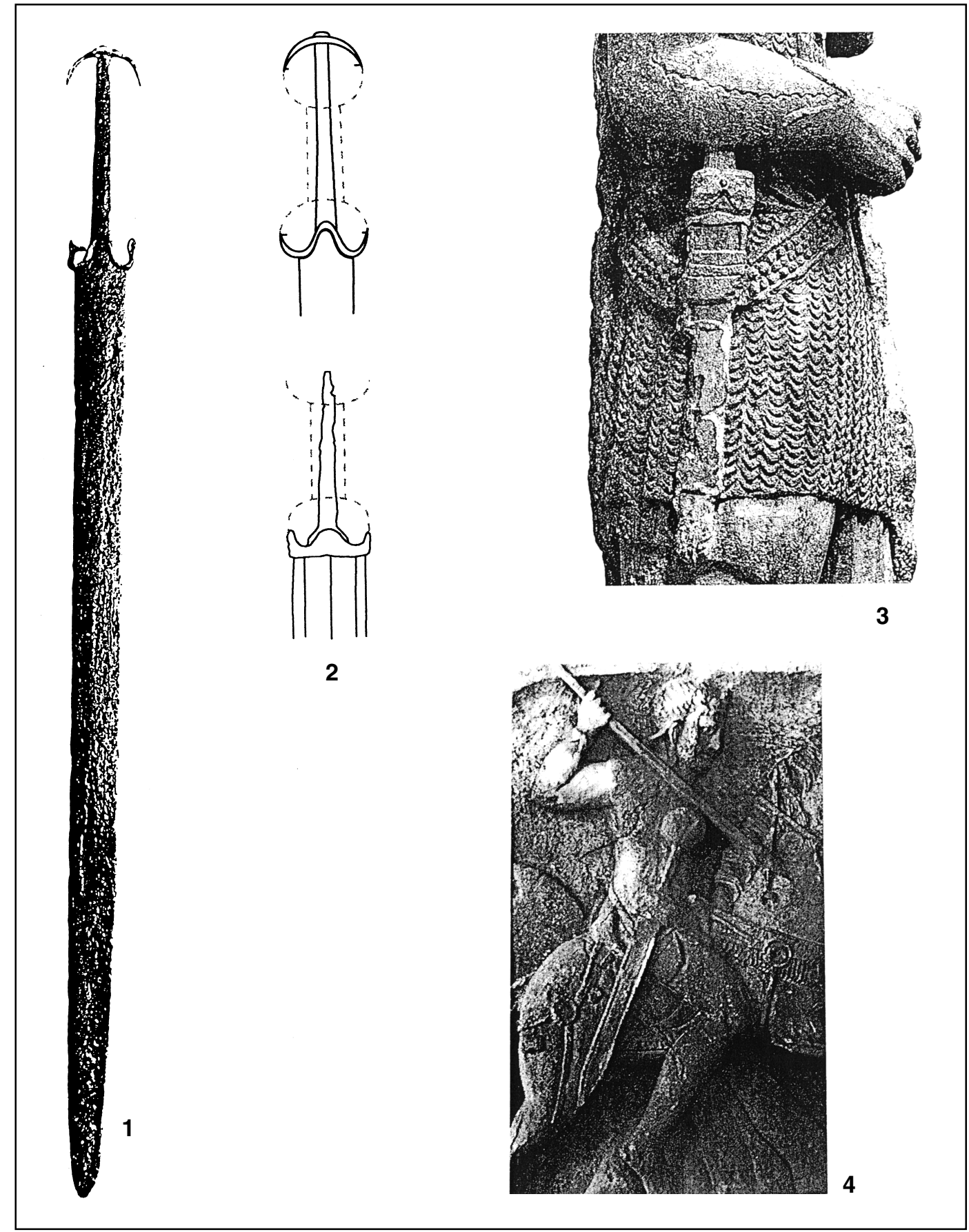

Pl. X. Spatha, gladius ou sabre gaulois: similitudes et différences.

1- épée gauloise provenant du site de La Tène (Suisse) avec pommeau et gardes hypertrophiées (II ${ }^{\mathrm{e}}$ s. av. J.-C.).

2- restitution de la poignée de l'épée gauloise de Pouilly-sur-Saône (cf. Pl. III) fin $\mathrm{II}^{\mathrm{e}}$ s. av. J.-C.).

3- détail de la poignée de l'épée de Vachères (Provence) ( $1^{\text {er }}$ s. av. J.-C.).

4- stèle de Flavius Bassus (Cologne) $\left(1^{\text {er }}\right.$ s. ap. J.-C.). 
L'ensemble de ces observations techniques et ornementales induit deux informations chronologiques.

- L'usage du bronze pour l'étui métallique laténien correspond à une technologie courante $\mathrm{au} \mathrm{V}^{\mathrm{e}}$ s. qui répond, dans un premier temps, aux nécessités de l'assemblage des gouttières latérales. Il devient progressivement obsolète pour disparaitre au début du IV ${ }^{\mathrm{e}}$ s., lorsque les problèmes techniques posés par l'élaboration des tôles de fer sont résolus. Pendant plus de deux siècles les fourreaux laténiens sont exclusivement en fer et l'utilisation minoritaire des tôles en alliages cuivreux ne recommence qu'à compter du milieu du $\mathrm{II}^{\mathrm{e}}$ s. Av. J.-C. avec certaines épées de l'horizon LT.D1.

- L'usage du chagrinage sur le droit des fourreaux de fer semble commencer au $\mathrm{III}^{\mathrm{e}} \mathrm{s}$. pour atteindre son apogée pendant la phase LT.C2 soit la première moitié du $\mathrm{II}^{\mathrm{e}}$ s. (De Navarro 1974). Le croisement de ces deux observations technologiques permet de situer l'élaboration du fourreau originel d'Alésia vers le tiers central du $\mathrm{II}^{\mathrm{e}} \mathrm{s}$.

- Un autre fragment de fourreau laténien en alliage cuivreux trouvé à Tiefenau, près de Berne, confirme cet horizon chronologique (Müller 1990). Son ornementation gravée sur l'entrée de l'étui correspond aussi à la fin de la phase LT.C2. Son découpage suivi de son enclouage sur un support le rapproche de l'usage secondaire, probablement votif, de l'exemplaire d'Alésia. Les traces de rituels semblables existent également sur des fourreaux du sanctuaire de Gournay-sur-Aronde (Lejars 1993, p. 219) ou en Espagne (Carme Rovira Hortala 1999).

En outre, l'enroulement de la tôle de bronze d'Alésia contient une information implicite tout à fait inattendue sur les fourreaux disparus de deux épées. En effet, cette «ligature» conserve l'empreinte d'un vide approximativement rectangulaire de 35 x $20 \mathrm{~mm}$ (Pl. VI, fig. 4). Un tel espace, nettement supérieur au volume des deux lames superposées permet de supposer la présence du matériau initial des deux étuis en bois qui n'ont laissé aucune trace directement identifiable. Les renforcements métalliques habituels des fourreaux en bois; entrées, gouttières, bouterolles et frettes de suspension, ont disparu, soit avant l'enfouissement des armes, soit au moment de leur exhumation au XIX ${ }^{\mathrm{e}} \mathrm{s}$.

Enfin, la tôle de fourreau enroulée porte les traces de coups de maillet portés du bas vers le haut comme pour bloquer, par pression, son enfoncement sur les fourreaux en bois tendre (Pl. VI, fig. 4). Ces dernières observations permettent d'émettre l'hypothèse d'un assemblage originel des deux armes dans leur étui de bois pour l'exposition éventuelle du petit trophée ainsi obtenu. L'oxydation du bois et son pourrissement progressif pourraient avoir nécessité d'enfoncer un peu plus leur «ligature» de bronze pour compenser la dégradation des étuis. Il est bien évidemment impossible d'estimer la durée de cet usage votif secondaire des deux gladii $^{7}$. Il semble cependant évident que la durée des campagnes de César, et encore moins celle du siège d'Alésia, ne fut pas suffisante pour entraîner un processus de dégradation demandant probablement plusieurs décennies.

Il n'est donc pas improbable que ces deux glaives, «prisonniers» d'une tôle de fourreau laténien, puissent être largement antérieurs à l'épisode militaire qui met fin à la Guerre des Gaules. La chronologie proposée pour l'élaboration des armes situerait la fabrication du trophée dans le dernier tiers du $\mathrm{II}^{\mathrm{e}}$ s. av. J.-C.

La finalité d'un tel usage votif pourrait correspondre à la commémoration d'un événement glorieux pour les gaulois du même type que celui de la défaite romaine par les Helvètes commandés par Divicone en 107 av. J.-C. César fait allusion au souvenir douloureux de la mort de Cassius et du passage de l'armée romaine sous le joug (BG livre 7,7).

7 Pour que les processus de minéralisation se produisent il faut que l'imprégnation des sels métalliques de la corrosion soit facilitée par le contact avec des matériaux organiques peu ou pas dégradés dans un milieu confiné. Dans le cas présent l'absence de telles traces lisibles peut résulter d'une longue phase de dégradation à l'air libre précédant l'enfouissement (Rapin 1988, p. 109, fig. 53). L'excellente conservation partielle de la partie haute des deux épées permet d'émettre aussi une autre hypothèse : celle d'une incinération du trophée détruisant les restes organiques et entraînant la disparition des composantes métalliques des fourreaux, avant l'enfouissement de l'ensemble. 
Le petit trophée faisait-il partie des bagages de Vercingétorix ? L'exhibition de ces armes ligaturées aurait-elle servie à ranimer la flamme belliqueuse de l'armée assiégée? Selon Plutarque, César lui-même aurait été témoin de telles pratiques de consécration chez les Arvernes ${ }^{8}$. La perte du trophée son incinération et/ou son enfouissement dans ou à proximité d'un sanctuaire d'Alésia vers la fin du siège, serait compatible avec l'ancienneté relative des deux armes.

\section{CONCLUSION}

Si l'appartenance des deux épées d'Alésia au genre gladius est incontestable, leur chronologie d'élaboration reste encore à vérifier et à préciser. Malgré la convergence des indices diachroniques recueillis sur l'ensemble du petit trophée d'Alésia l'argument chronologique majeur repose, entre autre, sur l'analyse de l'ensemble de Giubiasco. Une campagne de restauration vigilante sur le mobilier métallique de ce cimetière en particulier sur celui de la tombe 119 devrait donner une meilleure assise temporelle à tout un groupe d'armes romaines républicaines sans contexte. L'amorce d'un corpus se manifeste déjà autour des épées d'Alésia et, même s'il est encore trop faible pour constituer une véritable base d'analyses, le processus ainsi déclenché devrait entraîner un renouvellement des observations et des problématiques sur ce thème.

Parmi celles-ci il est probable qu'à l'instar des armes de poing gauloises, il sera aussi difficile de cerner les standards d'un type unique de glaive républicain. Plusieurs modules d'épées de dimensions et de morphologies différentes devaient certainement coexister pour répondre aux contraintes de spécialisations contemporaines aussi éloignées que celles du fantassin léger ou du cavalier.

Dans l'immédiat, l'évolution de la connaissance de ces équipements passe par un accroissement de la qualité du corpus. Un développement indispensable des analyses radiographiques devrait à coup sûr entraîner une augmentation spectaculaire des données archéologiques. Pour la majorité des armes citées en comparaison ces compléments d'analyses conditionnent leur identification. Les réserves des musées, comme la littérature et les fouilles récentes offrent un potentiel largement sous-estimé.

En second lieu, en fonction de l'accroissement des corpus, les gains d'information permettront peut-être de formuler les problématiques concernant les interpénétrations des innovations techniques et militaires contemporaines en Europe moyenne comme dans les péninsules ibériques et italiques.

Enfin, pour surprenante qu'elle soit, la morphologie de ces glaives anciens reste cohérente avec ce que l'on sait de leur évolution à compter du $1^{\mathrm{er}} \mathrm{s}$. ap. J.-C. Les quelques indices diachroniques identifiés sur les gladii postérieurs à la guerre des Gaules font état d'une tendance à la diminution de la longueur de la lame et à l'atténuation de son profil pistiliforme (Feugère 1993, p. 99). L'extrapolation en amont d'une telle tendance est compatible avec la chronologie proposée pour les deux longues armes d'Alésia. Elles sont vraisemblablement républicaines et plus proches de Polybe que de César.

\footnotetext{
8 «... car les Arvernes montrèrent une épée courte suspendue dans un de leur sanctuaire en affirmant que c'est une dépouille prise sur César. Il l'a dit lui-même par la suite et ne fit qu'en sourire. Comme ses amis l'engagèrent à l'enlever, il refusa, disant que l'objet était consacré à la divinité» (Plutarque, Vies parallèles, César, chap. 26, paragraphe 8, coll. Budé).
} 


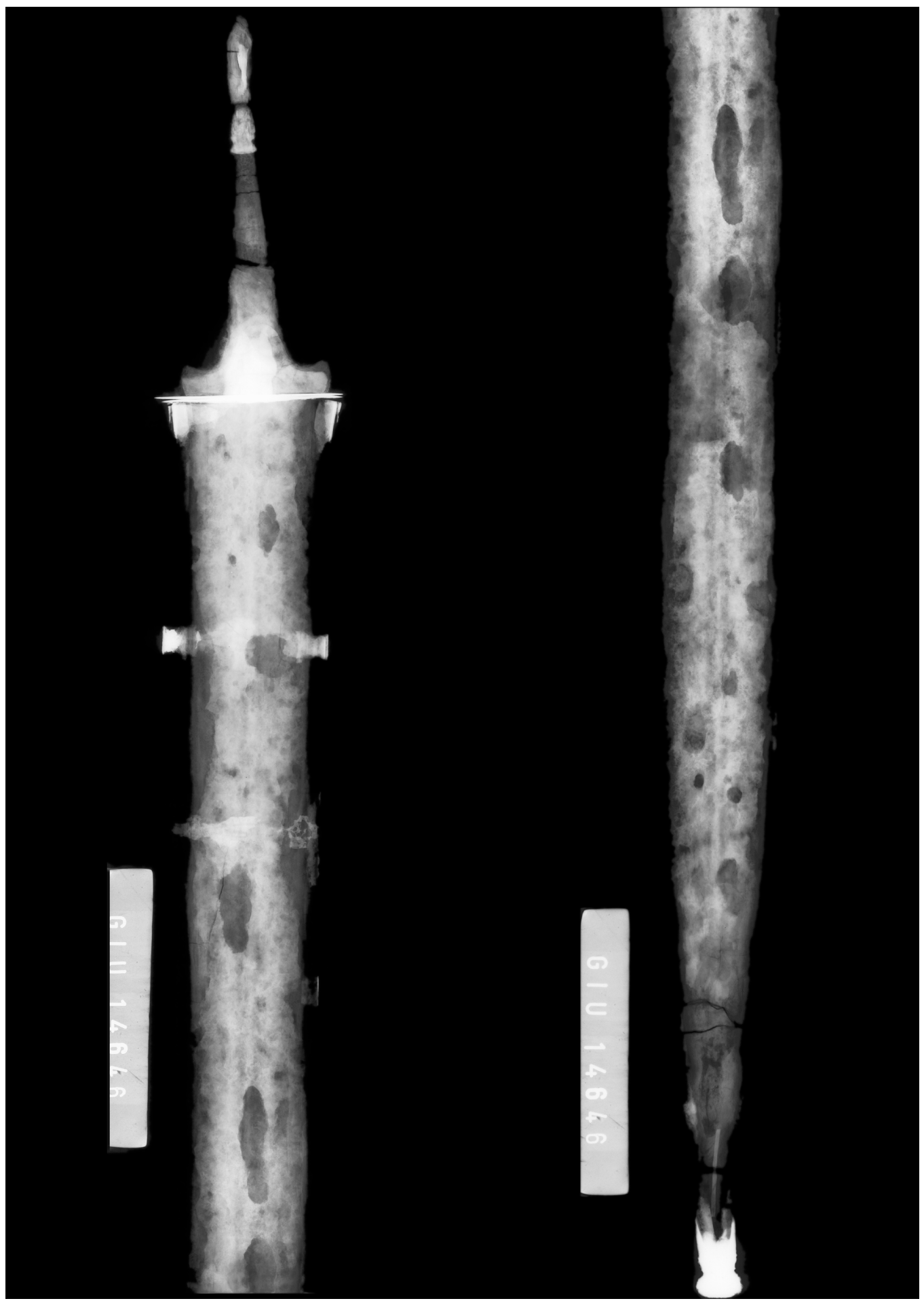

Cliché RX de la partie supérieure du glaive de la tombe 119 de Giubiasco (Musée National Suisse de Zurich) qui permet de verifier la présence de l'applique métallique campaniforme sur la garde de la poigneé. Sur le haut du cliché le remontage inversé de la soie est visible et, vers le bas, l'entrée du fourreau avec sa contreplaque horizontale est semblable à celle d'Alesia. 
REMERCIEMENTS

À Madame Hélene Chew, conservateur au Museé des Antiquités Nationales, Mousieur Philippe Della Casa, conservateur du Museé National Suisse, Mousieur Gilbert Kaenel du Museé Cantonal d'Archeologie de Lausane et Mousieur Christian Goudineau du Collège de France.

ANDRÉ RAPIN

Institut de Restauration et de Recherches Archéologiques et Paléométallurgiques (IRRAP), Compiegne

E-mail: irrap@aol.com

\section{BIBLIOGRAPHIE}

Abréviation: J.R.M.E.S Journal of Roman Military Equipment Studies. R.O.M.E.C Roman military Equipment Conference.

BARRUOl, G.; SAuZAde, G. (1969): Une tombe de guerrier à Saint-Laurent-des-Arbres (Gard). Contribution à l'étude des sépultures du $1^{\mathrm{er}} \mathrm{s}$. av. J.-C. dans la basse vallée du Rhone dans Revue d'Etudes Ligures XXXV, fig. 23, p. 43.

Bonnamour, Louis (1990, 1991): Du silex à la poudre... Catalogue de l'exposition 4000 ans d'armement en Val-de-Saône, ed. M. Mergoil, 1990, chapitre 4. Les armes romaines, par Michel Feugère, p. 93-97 et notice 112 .

Rovira Hortalà, Carme (1999): Las armas - trofeo en la cultura ibérica: pautas de identificación e interpretación, dans Gladius, T. XIX, Madrid 1999, p. 13-32.

CHEw, Hélène (1991,1992): Masques de fer. Un officier romain du temps de Caligula. Catalogue de l'exposition du Musée des Antiquités Nationales (p. 47).

Connoly, Peter (1997): Pilum, Gladius and Pugio in the Late Republic, dans J.R.M.E.S 8. Actes du $10^{\text {ème }}$ congrès international R.O.M.E.C tenu à Montpellier en 1996 sur le thème de l'équipement militaire et l'armement de la République (IV $-1^{\mathrm{er}}$ s. Av. J.-C.) sous la direction de M. Feugère (p. 41-47).

COUISsin, Paul (1926): Les armes romaines. Essai sur les origines et l'évolution des armes individuelles du légionnaire romain, Paris, 1926, p. 297-301, 311.

Dechelette, Joseph (1927): Manuel d'Archéologie Préhistorique et Celtique, IV, Picard, Paris1910, réédition 1927, p. 467 et 622, fig. 401 et 460.

DE NAVARro, José-Maria (1972): The Finds from the site of La Tène, II, London, Pl. LXXIX à LXXXIII et P1. XCV.

Duval, Alain (1994): Les découvertes effectuées lors des fouilles napoléniennes, catalogue de l'exposition Vercingétorix et Alésia; p. 263.

EGG, Markus (2000): Die Waffen der «Lepontier», dans, I Leponti, tra mito e realtà, 1, Gruppo Archeologia Ticino, Locarno, 2000, p. 319-339.

FEUGERE, Michel (1993): Les armes des Romains, Paris, Errance 1993.

Horvat, Jana (1990): Nauportus (Vrnika) dans Academia Scientiarium et Artium Slovenia, Ljubljana 1990, Pl. 27 et 28.

HoRvat, Jana (1997): Roman Republican Weapons from Smihel in Slovenia, dans J.R.M.E.S 8, p. $105-120$.

LEJARS Thierry (1993): Gournay III, Les fourreaux d'épée; Errance, Paris 1993, p. 219.

Metzler, J.; Waringo R.; Bis, R.; Metzler-Zens, N. (1991): Clémency et les tombes de l'aristocratie en Gaule Belgique, dans Dossiers d'Archéologie du Musée National d'Histoire et d'Art, I, Luxembourg 1991 , p. 113-114-115. 
PRIMAS, Margarita (1992): Grab 119 von Giubiasco und die Romanisierung der Poebene, dans Festschrift zum 50 jährigen Bestehen des Institutes fur Ur-und Fruhgeschichte der Leopold - Franzens-Universität, 8, Insbruck, 1992, p. 473-483.

QUESADA SANZ, Fernando (1997a): El armamento ibérico. Estudio tipológico, geográfico, functional, social y symbólico de las armas en la cultura ibérica (siglos VI-I a-c), dans Monographies Instrumentum, 3/1 ed. Monique Mergoil, Montagnac 1997.

QUESADA SANZ, Fernando (1997b):Gladius Hispaniensis: an archaelogical view from Iberia, dans JRMES, 8, p. 251-270.

RAPIN, André (1988): Gournay II, Boucliers et lances dans Revue Archéologique de Picardie, Errance, Paris, 1988, p. 71, fig. 38, 4-5-6.

RAPIN, André (1991): Le ceinturon métallique et l'évolution de la panoplie celtique au III ${ }^{\mathrm{e}} \mathrm{s}$. av. J.-C., dans Etudes celtiques XXVIII, CNRS, Paris 1991, p. 349-368.

RAPIN, André (1999): L'armement celtique en Europe: chronologie de son évolution technologique du V $\mathrm{V}^{\mathrm{e}}$ au $1^{\mathrm{er}}$ s. av. J.-C., dans Gladius XIX, Madrid 1999, fig. 7-8-9-10-11.

SCHAAFF, Ulrich (1984): Studien zen Keltischen Bewaffnung, dans Jahrbuch des RömischGermanischen Zentralmuseums Mainz 31, Mainz 1984, p. 623, fig. 11-12-13-14.

SCHÜLE, Wilhelm (1969): Die Meseta-Kulturen der iberischen Habinsel, Berlin 1969, P1. 64.

SIEVERS, Suzanne (1994): Les armes d'Alésia, dans Vercingetorix et Alésia, catalogue de l'exposition du Musée des Antiquités Nationales édité par la Réunion des Musées Nationaux, Paris 1994, p. 270.

SIEVERS, Suzanne; (1996): Armes Celtiques, germaniques et romaines: ce que nous apprennent les fouilles d'Alésia, dans L'armée romaine en Gaule, sous la direction de M. Reddé, Paris, ed. Errance, p. 67-81.

SIEVERS, Suzanne (1997): Alésia und Osuna: Bemerkungen zur Normierung der spätrepublikanischen Bewafnung und Ausrüstung, J.R.M.E.S 8, p. 271-276.

VERCHERE de REFFYE (1864): Les armes d'Alise dans Revue Archéologique, nouvelle série, $5^{\text {ème }}$ année, $10^{\text {ème }}$ volume, Paris 1864, p. 337-349, Pl. XXII, fig. 19.

VougA, Paul (1923): La Tène: Monographie de la station.., Leipzig 1923, Pl. III n 7 . La poignée de la même épée a été publiée antérieurement par VOUGA Emile, Les Helvètes, notice sur La Tène et son époque en Suisse, 1885, Pl. VIII, fig. 2. 